TRANSACTIONS OF THE

AMERICAN MATHEMATICAL SOCIETY

Volume 351, Number 2, February 1999, Pages 619-646

S 0002-9947(99)02186-8

\title{
GAP ESTIMATES \\ OF THE SPECTRUM OF HILL'S EQUATION AND ACTION VARIABLES FOR KdV
}

\author{
T. KAPPELER AND B. MITYAGIN
}

Abstract. Consider the Schrödinger equation $-y^{\prime \prime}+V y=\lambda y$ for a potential $V$ of period 1 in the weighted Sobolev space $\left(N \in \mathbb{Z}_{\geq 0}, \omega \in \mathbb{R}_{\geq 0}\right)$

$$
H^{N, \omega}\left(S^{1} ; \mathbb{C}\right):=\left\{f(x)=\sum_{k=-\infty}^{\infty} \hat{\hat{f}}(k) e^{i 2 \pi k x} \mid\|f\|_{N, \omega}<\infty\right\}
$$

where $\hat{\hat{f}}(k)(k \in \mathbb{Z})$ denote the Fourier coefficients of $f$ when considered as a function of period 1 ,

$$
\|f\|_{N, \omega}:=\left(\sum_{k}(1+|k|)^{2 N} e^{2 \omega|k|}|\hat{\hat{f}}(k)|^{2}\right)^{1 / 2}<\infty,
$$

and where $S^{1}$ is the circle of length 1 . Denote by $\lambda_{k} \equiv \lambda_{k}(V)(k \geq 0)$ the periodic eigenvalues of $-\frac{d^{2}}{d x^{2}}+V$ when considered on the interval $[0,2]$, with multiplicities and ordered so that $\operatorname{Re} \lambda_{j} \leq \operatorname{Re} \lambda_{j+1}(j \geq 0)$. We prove the following result.

Theorem. For any bounded set $\mathcal{B} \subseteq H^{N, \omega}\left(S^{1} ; \mathbb{C}\right)$, there exist $n_{0} \geq 1$ and $M \geq 1$ so that for $k \geq n_{0}$ and $V \in \mathcal{B}$, the eigenvalues $\lambda_{2 k}, \lambda_{2 k-1}$ are isolated pairs, satisfying (with $\left\{\lambda_{2 k}, \lambda_{2 k-1}\right\}=\left\{\lambda_{k}^{+}, \lambda_{k}^{-}\right\}$)

(i) $\sum_{k \geq n_{0}}(1+k)^{2 N} e^{2 \omega k}\left|\lambda_{k}^{+}-\lambda_{k}^{-}\right|^{2} \leq M$,

(ii) $\sum_{k \geq n_{0}}(1+k)^{2 N+1} e^{2 \omega k}\left|\left(\lambda_{k}^{+}-\lambda_{k}^{-}\right)-2 \sqrt{\hat{\hat{V}}(k) \hat{\hat{V}}(-k)}\right|^{2} \leq M$.

\section{InTRODUCTION AND SUMMARY OF THE RESULtS}

The Korteweg-deVries equation $(\mathrm{KdV})$ on the circle

$$
\partial_{t} U(x, t)=-\partial_{x}^{3} U(x, t)+6 U(x, t) \partial_{x} U(x, t)
$$

is a completely integrable Hamiltonian system of infinite dimension. We choose as its phase space the Sobolev space $H^{N, \omega}\left(S^{1}\right)$, where $S^{1}$ is the circle of length $1, \omega \in \mathbb{R}_{\geq 0}$ and $N \in \mathbb{Z}_{\geq 0}$. The Poisson structure is the one proposed by Gardner,

$$
\left\{F_{1}, F_{2}\right\}_{G}:=\int_{S^{1}} \frac{\partial F_{1}}{\partial V(x)} \frac{d}{d x} \frac{\partial F_{2}}{\partial V(x)} d x,
$$

where $F_{1}$ and $F_{2}$ are $C^{1}$ functionals on $H^{N, \omega}\left(S^{1}\right)$ and $\frac{\partial F}{\partial V(x)}$ denotes the $L^{2}$-gradient of $F$. The Gardner bracket is degenerate. Its symplectic leaves are given by

Received by the editors December 5, 1996.

1991 Mathematics Subject Classification. Primary 58F19, 58F07, 35Q35.

(C)1999 American Mathematical Society 


$$
\begin{aligned}
& H_{c}^{N, \omega}\left(S^{1}\right):=c+H_{0}^{N, \omega}\left(S^{1}\right) \text { with } c \in \mathbb{R} \text {, where } \\
& \qquad H_{0}^{N, \omega}\left(S^{1}\right):=\left\{f \in H^{N, \omega}\left(S^{1}\right) \mid \int_{S^{1}} f d x=0\right\} .
\end{aligned}
$$

Also we introduce the following weighted $l^{2}$-spaces:

$$
l_{N+\frac{1}{2}, \omega}^{2}\left(\mathbb{N} ; \mathbb{R}^{2}\right):=\left\{(x, y)=\left(x_{j}, y_{j}\right)_{j \geq 1} \mid \sum_{j \geq 1} j^{2 N+1} e^{2 \omega j}\left(x_{j}^{2}+y_{j}^{2}\right)<\infty\right\} .
$$

In section 3 we prove the following result.

Theorem 1. Let $N \in \mathbb{Z}_{\geq 0}$ and $\omega \in \mathbb{R}_{\geq 0}$. Then there exists a map

$$
\Lambda^{(N, \omega)}: H_{0}^{N, \omega}\left(S^{1}\right) \rightarrow l_{N+\frac{1}{2}, \omega}^{2}\left(N ; \mathbb{R}^{2}\right)
$$

with the following properties:

(1) $\Lambda^{(N, \omega)}$ is a diffeomorphism;

(2) $\Lambda^{(N, \omega)}$ and $\left(\Lambda^{(N, \omega)}\right)^{-1}$ are real analytic;

(3) the variables $\left(x_{j}^{2}+y_{j}^{2}\right) / 2$ associated to $\Lambda^{(N, \omega)}(V)=\left(x_{j}(V), y_{j}(V)\right)_{j \geq 1}$ are action coordinates of $K d V$ and its entire hierarchy.

We refer to $\left(x_{j}(V), y_{j}(V)\right)_{j \geq 1}$ as Birkhoff coordinates of KdV (and its hierarchy). The map, associating to $V$ Birkhoff coordinates, is referred to as Birkhoff map, and can be thought of as a nonlinear Fourier transform. Clearly, the Fourier transform $\mathcal{F}$ establishes a linear isomorphism between $H_{0}^{N, \omega}\left(S^{1}\right)$ and $l_{N, \omega}^{2}\left(\mathbb{N} ; \mathbb{R}^{2}\right), \mathcal{F}(V)=$ $(\operatorname{Re} \hat{\hat{V}}(k), \operatorname{Im} \hat{\hat{V}}(k))_{k \geq 1}$, and Theorem 1 is an instance of (probably) many properties Fourier transform and Birkhoff map have in common. Theorem 1 has already been established in the case $\omega=0$ [BKM1] (cf. also [Ka], [BBGK]). In order to prove that $\Lambda^{(N, \omega)}$ can be chosen as the restriction of $\Lambda$ to $H_{0}^{N, \omega}$, one has to derive asymptotic estimates for the periodic eigenvalues of the Schrödinger operator $L:=-\frac{d^{2}}{d x^{2}}+V$ for $V$ in $H_{0}^{N, \omega}\left(S^{1} ; \mathbb{C}\right)$ considered on the interval [0,2]. The periodic spectrum of $L$ is discrete. Denote it by $\left(\lambda_{k}=\lambda_{k}(V)\right)_{k \geq 0}$ (with multiplicities), where the $\lambda_{k}$ 's are ordered in such a way that $\operatorname{Re} \lambda_{0} \leq \operatorname{Re} \bar{\lambda}_{1} \leq \ldots$ and, in case $\operatorname{Re}\left(\lambda_{k}\right)=\operatorname{Re}\left(\lambda_{k+1}\right)$, $\operatorname{Im} \lambda_{k} \leq \operatorname{Im} \lambda_{k+1}$. For $k$ sufficiently large, the eigenvalues come in isolated pairs $\left\{\lambda_{2 k}, \lambda_{2 k-1}\right\}$. The main result of this paper is the following one, proved in section 2 :

Theorem 2. Let $\mathcal{B} \subseteq H^{N, \omega}\left(S^{1} ; \mathbb{C}\right)$ be a bounded set of potentials $\left(N \in \mathbb{Z}_{\geq 0}, \omega \in\right.$ $\left.\mathbb{R}_{\geq 0}\right)$. Then there exists $n_{0} \geq 1$ such that

$$
\sup _{V \in \mathcal{B}} \sum_{k \geq n_{0}}(1+k)^{2 N+1} e^{2 \omega k}\left|\lambda_{k}^{+}(V)-\lambda_{k}^{-}(V)-2 \sqrt{\hat{\hat{V}}(k) \hat{\hat{V}}(-k)}\right|^{2}<\infty,
$$

where $\left\{\lambda_{k}^{+}, \lambda_{k}^{-}\right\}=\left\{\lambda_{2 k}, \lambda_{2 k-1}\right\}$ (cf. Theorem 2.10 for the indexing $\lambda_{k}^{+}, \lambda_{k}^{-}$of the numbers $\left.\lambda_{2 k}, \lambda_{2 k-1}\right)$.

As a consequence of Theorem 2 one obtains

Corollary 3. For a real valued potential $V \in L^{2}\left(S^{1}\right)$ to be an element of $H^{N, \omega}\left(S^{1}\right)$ it is necessary and sufficient that

$$
\sum_{k=1}^{\infty}(1+k)^{2 N} e^{2 \omega k}\left(\lambda_{2 k}(V)-\lambda_{2 k-1}(V)\right)^{2}<\infty .
$$


In the case $\omega=0$, Theorem 2 and Corollary 3 have been established by Marčenko [Ma] by different methods which might, however, not be adaptable to the case $\omega>0$.

Theorem 1 can be used to prove by the 'inverse scattering method' that the Korteweg-deVries equation (1.1) is well-posed on the circle. To simplify the wording of the statement we restrict ourselves to the case where the initial data $V$ is in $H_{0}^{N, \omega}\left(S^{1}\right)$ (cf. [BKM2] in case $V$ has nonzero average).

Corollary 4. Let $N \in \mathbb{Z}_{\geq 0}$ and $\omega \in \mathbb{R}_{\geq 0}$. There exists a solution operator $\mathcal{S}$ : $H_{0}^{N, \omega}\left(S^{1}\right) \rightarrow C\left(\mathbb{R} ; H_{0}^{N, \omega}\left(S^{1}\right)\right)$ of (1.1) with the following properties:

(i) Given $V_{1}, V_{2}$ in $H_{0}^{N, \omega}\left(S^{1}\right)$, there exists $M>0$ so that for any $t \in \mathbb{R}$

$$
\left\|\mathcal{S}\left(V_{1}\right)(t)-\mathcal{S}\left(V_{2}\right)(t)\right\|_{H_{0}^{N, \omega}\left(S^{1}\right)} \leq M(1+|t|)\left\|V_{1}-V_{2}\right\|_{H_{0}^{N, \omega}\left(S^{1}\right)} .
$$

(ii) For any $0<T<\infty, \mathcal{S}: H_{0}^{N, \omega}\left(S^{1}\right) \rightarrow C\left([-T, T] ; H_{0}^{N, \omega}\left(S^{1}\right)\right)$ is real analytic.

Proof. The case $\omega=0, N=0$ can be treated as in [BKM2] (cf. also [Bo]). The same proof works for this more general situation. In fact, the case $\omega=0, N \geq 1$ or $\omega>0, N \geq 0$ is somewhat easier, as the frequencies of the KdV Hamiltonian are easily seen to be real analytic in these cases.

Remark. Results similiar to the one presented for KdV hold for any of the equations in the KdV hierarchy.

\section{Proof of Theorem 2}

In this section, we prove Theorem 2, stated in the introduction. First let us introduce some more notation.

Definition. $w:=(w(k))_{k \in \mathbb{Z}}$ is said to be a weight if

(i) $w(k) \geq 1(k \in \mathbb{Z})$;

(ii) there exists $M_{w} \geq 1$ such that $w(k) \leq M_{w} w(k-j) w(j) \quad(k, j \in \mathbb{Z})$.

Condition (ii) is refered to as the submultiplicative property of a weight.

Most frequently we will use the weight

$$
w(k):=\left(1+\left|\frac{k}{2}\right|\right)^{N} \quad e^{\frac{\omega}{2}|k|},
$$

where $N \in \mathbb{Z}_{\geq 0}$ and $\omega \in \mathbb{R}_{\geq 0}$. In that case, one can choose $M_{w}=1$ in condition (ii) of the above definition. The reason for choosing $\frac{\omega}{2}$ rather than $\omega$ in (2.1) follows from the observation that

$$
V=\sum_{k} \hat{\hat{V}}(k) e^{i 2 \pi k x}=\sum_{k} \hat{V}(2 k) e^{i \pi(2 k) x}
$$

for $V \in H^{N, \omega}\left(S^{1} ; \mathbb{C}\right)$, with $(\hat{V}(k))_{k \in \mathbb{Z}}$ denoting the Fourier coefficients of $V$ considered as a function of period 2 and thus

$$
\|V\|_{N, \omega}^{2}=\sum_{k}(1+|k|)^{2 N} e^{2 \omega|k|}|\hat{\hat{V}}(k)|^{2}=\sum_{k}\left(\left(1+\frac{|k|}{2}\right)^{N} e^{\frac{\omega}{2}|k|}\right)^{2}|\hat{V}(k)|^{2} .
$$

For $K \subseteq \mathbb{Z}$ and a weight $w$ denote by $l_{w}(K)$ the complex Hilbert space $l_{w}^{2}(K) \equiv$ $l_{w}^{2}(K ; \mathbb{C})$,

$$
l_{w}^{2}(K):=\left\{(a(k))_{k \in K} \mid\|a\|_{w}<\infty\right\}
$$


where

$$
\|a\|_{w} \equiv\|a\|_{l_{w}^{2}(K)}:=\left(\sum_{k \in K} w(k)^{2}|a(k)|^{2}\right)^{1 / 2} .
$$

Most frequently, we will use for $K$ the set $\mathbb{Z}$ or $\mathbb{Z}(n):=\mathbb{Z} \backslash\{ \pm n\}$. If necessary for clarity, we will sometimes write $a_{K}$ for a sequence $(a(k))_{k \in K} \in l_{w}^{2}(K)$.

For a linear operator $A: l_{w_{1}}^{2}\left(K_{1}\right) \rightarrow l_{w_{2}}^{2}\left(K_{2}\right)$ we denote by $A(k, j)$ its matrix elements

$$
(A a)(k):=\sum_{j \in K_{1}} A(k, j) a(j) \quad\left(k \in K_{2}\right) .
$$

Definition. $\mathcal{S}: l^{2}(\mathbb{Z}) \rightarrow l^{2}(\mathbb{Z})$ is defined by $(\mathcal{S} a)(k):=a(k+1)(k \in \mathbb{Z})$. $\mathcal{S}$ is called the shift operator. The restriction of $\mathcal{S}$ to $l_{w}^{2}(K)$ with values in $l_{\mathcal{S} w}^{2}(K)$ is denoted by $\mathcal{S}$ as well, and $\mathcal{S}^{n}=\mathcal{S} \circ \cdots \circ \mathcal{S}$ denotes the $n$th iterate of $\mathcal{S}$. Notice that

$$
\begin{aligned}
\left\|\mathcal{S}^{n} a\right\|_{l_{\mathcal{S}^{n} w}^{2}(K)}^{2} & =\sum_{k \in K}\left(\mathcal{S}^{n} w\right)(k)^{2}\left|\left(\mathcal{S}^{n} a\right)(k)\right|^{2} \\
& =\sum_{k \in K} w(k+n)^{2}|a(k+n)|^{2} \leq\|a\|_{l_{w}^{2}(\mathbb{Z})}^{2} .
\end{aligned}
$$

Definition. $\mathcal{J}: l^{2}(\mathbb{Z}) \rightarrow l^{2}(\mathbb{Z})$ is the involution given by

$$
(\mathcal{J} a)(k):=a(-k) \text {. }
$$

To prove Theorem 2, it suffices to consider potentials $V \in H_{0}^{N, \omega}\left(S^{1}\right)$ (where $S^{1}$ is the circle of unit length), as adding a constant $c$ to $V$ simply shifts the periodic spectrum of $-\frac{d^{2}}{d x^{2}}+V$ by $c$.

Express $-\frac{d^{2}}{d x^{2}}+V-\lambda$, acting on functions periodic of period 2, in Fourier space, as $A: l^{2}(\mathbb{Z}) \rightarrow l^{2}(\mathbb{Z})$, with

$$
A(k, j)=\pi^{2} k^{2} \delta_{k j}+\hat{V}(k-j) .
$$

Recall that $\hat{V}(0)=\frac{1}{2} \int_{0}^{2} V(x) d x=0$, as $V \in H_{0}^{N, \omega}\left(S^{1} ; \mathbb{C}\right)$, and that $(\hat{V}(k))_{k \in \mathbb{Z}}$ denote the Fourier coefficients of $V$ when considered as functions of period 2.

To analyze the eigenvalues $\lambda_{2 n}, \lambda_{2 n-1}$ near $n^{2} \pi^{2}(n \geq 1)$, write $\lambda=n^{2} \pi^{2}+z$. Writing $l^{2}(\mathbb{Z})$ as a direct sum $l^{2}(\mathbb{Z})=\mathbb{C} \oplus \mathbb{C} \oplus \mathbb{Z}(n), a=\left(a(-n), a(n), a_{\mathbb{Z}(n)}\right)$ $(\mathbb{Z}(n):=\mathbb{Z} \backslash\{ \pm n\})$, we see that $A-\lambda$ is of the form

$$
\begin{aligned}
& \left(((A-\lambda)(k,-n))_{k \in \mathbb{Z}} \quad((A-\lambda)(k, n))_{k \in \mathbb{Z}}((A-\lambda)(k, j))_{\substack{k \in \mathbb{Z} \\
j \in \mathbb{Z}(n)}}\right) \\
& =\left(\begin{array}{lll}
-z & \hat{V}(-2 n) & \left(\mathcal{S}^{n} \mathcal{J} \hat{V}\right)_{\mathbb{Z}(n)}^{T} \\
\hat{V}(2 n) & -z & \left(\mathcal{S}^{-n} \mathcal{J} \hat{V}\right)_{\mathbb{Z}(n)}^{T} \\
\left(\mathcal{S}^{n} \hat{V}\right)_{\mathbb{Z}(n)} & \left(\mathcal{S}^{-n} \hat{V}\right)_{\mathbb{Z}(n)} & B_{n}-z
\end{array}\right),
\end{aligned}
$$

where the superscript $T$ denotes the transpose and where $B_{n}: l^{2}(\mathbb{Z}(n)) \rightarrow l^{2}(\mathbb{Z}(n))$ is given by

$$
\begin{aligned}
& B_{n}:=A_{n}-\pi^{2} n^{2} I d_{n}, \\
& A_{n}:=\left(A_{n}(j, k)\right)_{j, k \in \mathbb{Z}(n)} .
\end{aligned}
$$


The (possibly) complex number $\lambda=n^{2} \pi^{2}+z$ is a periodic eigenvalue for $-\frac{d^{2}}{d x^{2}}+V$ if there exists $a=(a(k))_{k \in \mathbb{Z}} \in l^{2}(\mathbb{Z})$ such that

$$
(A-\lambda) a=0 .
$$

With $x:=a(-n), y:=a(n)$, this equation can be written as a system of three equations:

$$
\begin{gathered}
-z x+\hat{V}(-2 n) y+\left\langle\mathcal{S}^{n} \mathcal{J} \hat{V}, a_{\mathbb{Z}(n)}\right\rangle=0, \\
\hat{V}(2 n) x-z y+\left\langle\mathcal{S}^{-n} \mathcal{J} \hat{V}, a_{\mathbb{Z}(n)}\right\rangle=0 \\
\left(\mathcal{S}^{n} \hat{V}\right)_{\mathbb{Z}(n)} x+\left(\mathcal{S}^{-n} \hat{V}\right)_{\mathbb{Z}(n)} y+\left(B_{n}-z\right) a_{\mathbb{Z}(n)}=0 .
\end{gathered}
$$

Here

$$
\langle a, b\rangle \equiv\langle a, b\rangle_{\mathbb{Z}(n)}=\sum_{k \in \mathbb{Z}(n)} a(k) b(k)
$$

(no complex conjugation). To solve (2.7) for $a_{\mathbb{Z}(n)}$ we need to analyze the operator

$$
z-B_{n}: l^{2}(\mathbb{Z}(n)) \rightarrow l^{2}(\mathbb{Z}(n))
$$

Denote by $B_{n}^{I}$ the diagonal part of $B_{n}$,

$$
B_{n}^{I}(k, j)=\pi^{2}\left(k^{2}-n^{2}\right) \delta_{k j} \quad(k, j \in \mathbb{Z}(n))
$$

and define $B_{n}^{I I}:=B_{n}-B_{n}^{I}$. Notice that, with $M \geq 10$, for any $n \geq \frac{M}{2}$ and $|z| \leq M$, $z-B_{n}^{I}$ is invertible. Denote by $\|V\|$ the norm of $V$ in $L^{2}\left(S^{1}\right)$, and introduce

$$
\begin{gathered}
T_{n}:=B_{n}^{I I}\left(z-B_{n}^{I}\right)^{-1}: l^{2}(\mathbb{Z}(n)) \rightarrow l^{2}(\mathbb{Z}(n)), \\
n_{0}:=\max \left(\frac{M+1}{2},\|V\|\right), \quad M \geq 10 .
\end{gathered}
$$

Lemma 2.1. For $n \geq n_{0}$ and $|z| \leq M$,

$$
\begin{aligned}
& \left\|\left|T_{n}\right|\right\|_{\mathcal{L}\left(l^{2}(\mathbb{Z}(n))\right)} \leq \frac{\|V\|}{5 n} \leq \frac{1}{5} ; \quad \|\left(z-B_{n}^{I}\right)^{-1}||_{\mathcal{L}\left(l^{2}(\mathbb{Z}(n))\right)} \leq \frac{1}{5 n} \\
& z-B_{n} \text { is invertible and } \|\left.\left|\left(z-B_{n}\right)^{-1}\right|\right|_{\mathcal{L}\left(l^{2}(\mathbb{Z}(n))\right)} \leq \frac{1}{4 n}
\end{aligned}
$$

Remark. The conditions in Lemma 2.1 (and subsequent lemmas) are only assumed to insure that the quantities involved are well defined.

Proof. To obtain estimates (2.11) notice that $\left\|\mid T_{n}\right\|\left\|_{\mathcal{L}\left(l^{2}(\mathbb{Z}(n))\right)} \leq\right\| T_{n} \|_{H S}$, where $\left\|T_{n}\right\|_{H S}$ denotes the Hilbert-Schmidt norm of $T_{n}$ :

$$
\begin{aligned}
\left\|T_{n}\right\|_{H S}^{2} & =\sum_{j, k \in \mathbb{Z}(n)} \frac{|\hat{V}(k-j)|^{2}}{\left|z-\pi^{2}\left(k^{2}-n^{2}\right)\right|^{2}} \leq\|V\|^{2} \sum_{k \neq \pm n} \frac{1}{\left|\pi^{2}\right| k^{2}-n^{2}|-M|^{2}} \\
& \leq \frac{\|V\|^{2}}{75} \sum_{k \neq \pm n} \frac{1}{\left|k^{2}-n^{2}\right|^{2}} \leq \frac{\pi^{2}}{300} \frac{\|V\|^{2}}{n^{2}}<\frac{\|V\|^{2}}{30 n^{2}}
\end{aligned}
$$

Similarly,

$$
\begin{gathered}
\|\|\left(z-B_{n}^{I}\right)^{-1} \mid\left\|_{\mathcal{L}\left(l^{2}(\mathbb{Z}(n))\right)}^{2} \leq\right\|\left(z-B_{n}^{I}\right)^{-1} \|_{H S}^{2} \\
\leq \sum_{k \neq \pm n} \frac{1}{\left|\pi^{2}\right| k^{2}-n^{2}|-M|^{2}} \leq \frac{1}{30 n^{2}}
\end{gathered}
$$


To prove (2.12), write

$z-B_{n}=z-B_{n}^{I}-B_{n}^{I I}=\left(I d_{n}-B_{n}^{I I}\left(z-B_{n}^{I}\right)^{-1}\right)\left(z-B_{n}^{I}\right)=\left(I d_{n}-T_{n}\right)\left(z-B_{n}^{I}\right)$.

Then $\left(z-B_{n}\right)^{-1}=\left(z-B_{n}^{I}\right)^{-1}\left(I d_{n}-T_{n}\right)^{-1}$ and

$$
\left\|\left(z-B_{n}\right)^{-1} \mid\right\|_{\mathcal{L}\left(l^{2}(\mathbb{Z}(n))\right.} \leq \frac{1}{5 n} \sum_{k \geq 0}\left\|T_{n}\right\|^{k} \leq \frac{1}{5 n} \frac{1}{1-\frac{1}{5}}=\frac{1}{4 n} .
$$

In view of Lemma $2.1,(2.7)$ can be solved for $a_{\mathbb{Z}(n)}$, if $n \geq n_{0}$ and $|z| \leq M$ :

$$
a_{\mathbb{Z}(n)}=\left(z-B_{n}\right)^{-1}\left(\mathcal{S}^{n} \hat{V}\right)_{\mathbb{Z}(n)} x+\left(z-B_{n}\right)^{-1}\left(\mathcal{S}^{-n} \hat{V}\right)_{\mathbb{Z}(n)} y .
$$

If this is substituted into (2.5) and (2.6), we obtain (with $B_{-n}:=B_{n}$ )

$$
\left(\begin{array}{cc}
-z+\alpha(-n, z) & \hat{V}(-2 n)+\beta(-n, z) \\
\hat{V}(2 n)+\beta(n, z) & -z+\alpha(n, z)
\end{array}\right)\left(\begin{array}{l}
x \\
y
\end{array}\right)=\left(\begin{array}{l}
0 \\
0
\end{array}\right)
$$

where

$$
\alpha(n, z):=\left\langle\mathcal{S}^{-n} \mathcal{J} \hat{V},\left(z-B_{n}\right)^{-1}\left(\mathcal{S}^{-n} \hat{V}\right)_{\mathbb{Z}(n)}\right\rangle
$$

and

$$
\beta(n, z):=\left\langle\mathcal{S}^{-n} \mathcal{J} \hat{V},\left(z-B_{n}\right)^{-1}\left(\mathcal{S}^{n} \hat{V}\right)_{\mathbb{Z}(n)}\right\rangle .
$$

To analyze (2.14) we begin by investigating $\alpha(n, z)$.

Lemma 2.2. For $n \geq n_{0}$ and $|z| \leq M$,

$$
\alpha(n, z)=\alpha(-n, z) .
$$

Proof. By Lemma 2.3 (iii) below, $\left(z-B_{n}\right)^{-1}(k, j)=\left(z-B_{n}\right)^{-1}(-j,-k)$ and thus

$$
\begin{aligned}
\alpha(n, z) & =\sum_{k, j \neq \pm n} \hat{V}(n-k)\left(z-B_{n}\right)^{-1}(k, j) \hat{V}(j-n) \\
& =\sum_{k, j \neq \pm n} \hat{V}(-k-(-n))\left(z-B_{n}\right)^{-1}(-j,-k) \hat{V}((-n)-(-j)) \\
& =\sum_{k^{\prime}, j^{\prime} \neq \pm n} \hat{V}\left((-n)-k^{\prime}\right)\left(z-B_{n}\right)^{-1}\left(k^{\prime}, j^{\prime}\right) \hat{V}\left(j^{\prime}-(-n)\right) \\
& =\alpha(-n, z) .
\end{aligned}
$$

Lemma 2.3. For $n \geq n_{0}$ and $j, k \in \mathbb{Z}(n)$,

(i) $A_{n}(k, j)=A_{n}(-j,-k)$,

(ii) $\left(A_{n}-\lambda\right)^{-1}(k, j)=\left(A_{n}-\lambda\right)^{-1}(-j,-k)$,

(iii) $\left(z-B_{n}\right)^{-1}(k, j)=\left(z-B_{n}\right)^{-1}(-j,-k)$.

Proof. (i) Recall that, for $k, j \in \mathbb{Z}(n), A_{n}(k, j)=k^{2} \pi^{2} \delta_{k j}+\hat{V}(k-j)$. Therefore $A_{n}(k, j)=(-k)^{2} \pi^{2} \delta_{(-k)(-j)}+\hat{V}((-j)-(-k))=A_{n}(-j,-k)$.

(ii) is a straightforward verification, and (iii) follows from (ii).

By Lemma 2.2, the system of equations (2.14) with $n \geq n_{0}$ has a nontrivial solution $\left(\begin{array}{l}x \\ y\end{array}\right)$ for some $|z| \leq M$, if there exists $z \in \mathbb{C},|z| \leq M$, such that

$$
(z-\alpha(n, z))^{2}-(\hat{V}(-2 n)+\beta(-n, z))(\hat{V}(2 n)+\beta(n, z))=0 .
$$


Equation (2.17) is solved in two steps:

$$
z_{n}=\alpha\left(n, z_{n}\right)+\zeta \quad\left(\zeta \text { in } \mathcal{D}_{\frac{M}{2}}:=\left\{\zeta \in \mathbb{C}|| \zeta \mid<\frac{M}{2}\right\}\right)
$$

and, with $z\left(\zeta_{n}\right):=z_{n}\left(\zeta_{n}\right)$ given by $(2.18)$,

$$
\zeta_{n}^{2}-\left(\hat{V}(-2 n)+\beta\left(-n, z\left(\zeta_{n}\right)\right)\right)\left(\hat{V}(2 n)+\beta\left(n, z\left(\zeta_{n}\right)\right)\right)=0 .
$$

Let us first discuss equation (2.18). To solve it, we use the contractive mapping principle. For that purpose, we need

Lemma 2.4. For $|z| \leq M$ and $n \geq n_{0}$,

(i) $|\alpha(n, z)| \leq \frac{1}{4 n}\|V\|^{2}$,

(ii) $\left|\frac{d}{d z} \alpha(n, z)\right| \leq \frac{1}{16 n^{2}}\|V\|^{2}$.

Proof. (i) By (2.12) of Lemma 2.1, for $n \geq n_{0}$ and $|z| \leq M$,

$$
|\alpha(n, z)| \leq\|V\|\left|\left\|( z - B _ { n } ) ^ { - 1 } \left|\|\mid\| V \| \leq \frac{\|V\|^{2}}{4 n} .\right.\right.\right.
$$

(ii) Notice that

$$
\frac{d}{d z} \alpha(n, z)=\left\langle\mathcal{S}^{-n} \mathcal{J} \hat{V},-\left(z-B_{n}\right)^{-2} \mathcal{S}^{-n} \hat{V}\right\rangle
$$

and therefore

$$
\left|\frac{d}{d z} \alpha(n, z)\right| \leq\|V\|\left|\left\|\left(z-B_{n}\right)^{-1} \mid\right\|^{2}\|V\| \leq \frac{\|V\|^{2}}{16 n^{2}} .\right.
$$

Introduce

$$
n_{1}:=\max \left(n_{0},\|V\|^{2}\right)
$$

Proposition 2.5. For $\zeta \in \mathcal{D}_{M / 2}$ and $n \geq n_{1}$, the equation

$$
z_{n}=\alpha\left(n, z_{n}\right)+\zeta
$$

has a unique solution $z_{n}=z_{n}(\zeta)$ in $\mathcal{D}_{M}$, which depends analytically on $\zeta \in \mathcal{D}_{\frac{M}{2}}$.

Proof. By Lemma 2.4, since $n \geq n_{1}$,

$$
|\alpha(n, z)| \leq \frac{\|V\|^{2}}{4 n} \leq \frac{1}{4} \quad\left(z \in \overline{\mathcal{D}}_{M}\right)
$$

Therefore, $F(z) \equiv F_{n, \zeta}(z):=\zeta+\alpha(n, z)$ defines a map $F: \overline{\mathcal{D}}_{M} \rightarrow \overline{\mathcal{D}}_{M}$ (use $|F(z)| \leq|\zeta|+|\alpha(n, z)| \leq \frac{M}{2}+\frac{1}{4}<M$ for $\left.M \geq 10\right)$. $F$ is a contraction, as for any pair $z_{1}, z_{2} \in \overline{\mathcal{D}}_{M}$ 


$$
\begin{gathered}
\left|F\left(z_{1}\right)-F\left(z_{2}\right)\right| \leq\left(\sup _{|z| \leq M}\left|\frac{d}{d z} \alpha(n, z)\right|\right)\left|z_{1}-z_{2}\right| \\
\leq \frac{1}{16 n}\left(\frac{1}{n}\|V\|^{2}\right) \cdot\left|z_{1}-z_{2}\right| \leq \frac{1}{n} \cdot \frac{1}{16} \cdot\left|z_{1}-z_{2}\right| \leq \frac{1}{2}\left|z_{1}-z_{2}\right| .
\end{gathered}
$$

Thus, for any $\zeta \in \mathcal{D}_{\frac{M}{2}}$ and $n \geq n_{1}, F$ admits a unique fixed point $z_{n}=z_{n}(\zeta)$, and $z_{n}(\zeta)$ depends analytically on $\zeta$.

It remains to consider (2.19), which requires an estimate of $\beta( \pm n, z)$. First we need some auxilary results. In (2.9) we introduced the operator

$$
T_{n}:=B_{n}^{I I}\left(z-B_{n}^{I}\right)^{-1} \in \mathcal{L}\left(l^{2}(\mathbb{Z}(n)) .\right.
$$

This operator can also be viewed as an element in $\mathcal{L}\left(l_{\mathcal{S}^{n} w}^{2}(\mathbb{Z}(n))\right)$, where $w=$ $(w(j))_{j \in \mathbb{Z}}$ is the weight $w(j):=\left(1+\left|\frac{j}{2}\right|\right)^{N} e^{\frac{\omega}{2}|j|}$ and $\left(\mathcal{S}^{n} w\right)(j):=w(j+n)$. Denote by $W_{n}: l_{\mathcal{S}^{n} w}^{2}(\mathbb{Z}(n)) \rightarrow l^{2}(\mathbb{Z}(n))$ the operator given by

$$
W_{n}(k, j):=w(k+n) \delta_{k j} .
$$

Notice that $W_{n}$ is an isometry. Therefore the operator norm of

$$
\tilde{T}_{n}:=W_{n} T_{n} W_{n}^{-1}
$$

is given by

$$
\left.||\left|\tilde{T}_{n}\right|\right|_{\mathcal{L}\left(l^{2}(\mathbb{Z}(n))\right)}=\||| T_{n}||_{\left.\mathcal{L}_{\left(l^{n} w\right.}^{2}(\mathbb{Z}(n))\right)} .
$$

Lemma 2.6. For $n \geq n_{0}$ and $|z| \leq M$

$$
\left\|\mid T_{n}\right\|_{\mathcal{L}\left(l_{\mathcal{S}^{n} w}^{2}(\mathbb{Z}(n))\right)} \leq \frac{\|V\|_{N, \omega}}{5 n} .
$$

Proof. In view of (2.23), it suffices to estimate the Hilbert Schmidt norm of $\tilde{T}_{n}$ in $\mathcal{L}\left(l^{2}(\mathbb{Z}(n))\right)$. It follows from the submultiplicative property of the weight $w$ (cf. the definition at the beginning of section 2$)$ that

$$
\frac{\left(\mathcal{S}^{n} w\right)(j)}{\left(\mathcal{S}^{n} w\right)(k)} \leq w(j-k) .
$$

Therefore

$$
\begin{aligned}
\left\|\tilde{T}_{n}\right\|_{H S}^{2} & =\sum_{j, k \neq \pm n} \frac{\left|\mathcal{S}^{n} w(j)\right|^{2}}{\left|\mathcal{S}^{n} w(k)\right|^{2}}|\hat{V}(j-k)|^{2} \frac{1}{\left|z-\pi^{2}\left(k^{2}-n^{2}\right)\right|^{2}} \\
& \leq \sum_{j, k \neq \pm n}|w(j-k)|^{2}|\hat{V}(j-k)|^{2} \frac{1}{75} \frac{1}{(k-n)^{2}(k+n)^{2}} \\
& \leq \frac{1}{75} \sum_{i}\left(|w(i) \hat{V}(i)|^{2} \sum_{j-i \neq \pm n} \frac{1}{\left((j-i)^{2}-n^{2}\right)^{2}}\right) \\
& \leq\|V\|_{N, \omega}^{2} \frac{1}{30 n^{2}},
\end{aligned}
$$

where, for the last inequality, we argue in the same way as in the last steps of the inequality (2.13).

Let

$$
n_{2}:=\max \left(n_{1},\|V\|_{N, \omega}\right)=\max \left(\frac{M+1}{2},\|V\|^{2},\|V\|_{N, \omega}\right) .
$$


Proposition 2.7. For $n \geq n_{2}$,

$$
\left(\sum_{n \geq n_{2}}\left(1+\frac{n}{2}\right)^{2 N+2} e^{2 \omega n} \sup _{|z| \leq M}|\beta( \pm n, z)|^{2}\right)^{1 / 2} \leq\|V\|_{N, \omega}^{2}\left(1+\frac{1}{8}\|V\|_{N, \omega}\right)
$$

$$
\left(\sum_{n \geq n_{2}}\left(1+\frac{n}{2}\right)^{2 N+4} e^{2 \omega n} \sup _{|z| \leq M}\left|\frac{d}{d z} \beta( \pm n, z)\right|^{2}\right)^{1 / 2} \leq \frac{1}{4}\|V\|_{N, \omega}^{2}\left(1+\|V\|_{N, \omega}\right) .
$$

Proof. The estimates for $\beta(n, z)$ and $\beta(-n, z)$ are obtained in the same way. Let us concentrate on $\beta(n, z)$.

(i) Proof of (2.27): By Lemma 2.6 and $(2.26), \quad\left(I d_{n}-T_{n}\right) \in \mathcal{L}\left(l_{\mathcal{S}^{n} w}^{2}(\mathbb{Z}(n))\right)$ is invertible for $n \geq n_{2}$. With

$$
\left(I d_{n}-T_{n}\right)^{-1}=I d_{n}+T_{n}\left(I d_{n}-T_{n}\right)^{-1}
$$

and $a_{n} \equiv a_{n}(z) \in l_{w}^{2}(\mathbb{Z}(n))$ defined by

$$
\mathcal{S}^{n} a_{n}:=\left(I d_{n}-T_{n}\right)^{-1} \mathcal{S}^{n} \hat{V} \in l_{\mathcal{S}^{n} w}^{2}(\mathbb{Z}(n))
$$

the expression $\beta(n, z)$ takes the form $\beta(n, z)=\beta_{1}(n, z)+\beta_{2}(n, z)$ with

$$
\begin{aligned}
& \beta_{1}(n, z):=\left\langle\mathcal{S}^{-n} \mathcal{J} \hat{V},\left(z-B_{n}^{I}\right)^{-1} \mathcal{S}^{n} \hat{V}\right\rangle, \\
& \beta_{2}(n, z):=\left\langle\mathcal{S}^{-n} \mathcal{J} \hat{V},\left(z-B_{n}^{I}\right)^{-1} T_{n} \mathcal{S}^{n} a_{n}\right\rangle .
\end{aligned}
$$

The two terms $\beta_{1}(n, z)$ and $\beta_{2}(n, z)$ are estimated separately: For $n \geq n_{2}$

$$
\begin{aligned}
\sup _{|z| \leq M}\left|\beta_{1}(n, z)\right| & \leq \sum_{k \neq \pm n}|\hat{V}(n-k)| \frac{1}{8\left|k^{2}-n^{2}\right|}|\hat{V}(n+k)| \\
& =\frac{1}{8}\left(b_{\hat{V}} * b_{\hat{V}}\right)(2 n),
\end{aligned}
$$

where

$$
b_{\hat{V}}(j):=\frac{|\hat{V}(j)|}{j}(j \neq 0) \quad \text { and } \quad b_{\hat{V}}(0):=0 .
$$

Notice that $\left\|b_{\hat{V}}\right\|_{N+1, \frac{\omega}{2}} \leq 2^{N+1}\|V\|_{N, \omega}$.

Using the fact that $\|a * b\| \leq\left(\sum_{k}|a(k)|\right)\|b\|$ for $(a(k))_{k \in \mathbb{Z}} \in l^{1}(\mathbb{Z})$ and $(b(k))_{k \in \mathbb{Z}} \in l^{2}(\mathbb{Z})$, we conclude that

$$
\begin{aligned}
\sum_{n \geq n_{2}} & \left(1+\frac{n}{2}\right)^{2 N+2} e^{2 \omega n} \sup _{|z| \leq M}\left|\beta_{1}(n, z)\right| \\
\leq & \frac{1}{64} \sum_{n \neq 0}\left|\left(b_{\hat{V}} * b_{\hat{V}}\right)(2 n)\right|^{2}\left(1+\frac{|n|}{2}\right)^{2 N+2} e^{\omega(2|n|)} \\
\leq & \frac{1}{64} \pi^{2}\left(2\|V\|_{N, \omega}\right)^{4}=\left(\frac{\pi}{2}\|V\|_{N, \omega}^{2}\right)^{2}
\end{aligned}
$$


Direct computations furnish a slightly better estimate:

$$
\begin{aligned}
& \left(\sum_{n \geq n_{2}}\left(1+\frac{n}{2}\right)^{2 N+2} e^{2 \omega n} \sup _{|z| \leq M}\left|\beta_{1}(n, z)\right|^{2}\right)^{1 / 2} \\
& \quad \leq \frac{1}{4}\left(\sum_{j \neq 0} \frac{e^{\frac{\omega}{2}|j|}|\hat{V}(j)|}{|j|}+\sum_{j \neq 0} \frac{e^{\frac{\omega}{2}|j|}|\hat{V}(j)|}{|j|}\right)\|V\|_{N, \omega} \\
& \quad \leq \frac{1}{2}\left(\sum_{j \neq 0} \frac{1}{j^{2}}\right)^{1 / 2}\|V\|_{N, \omega}^{2} \leq\|V\|_{N, \omega}^{2} .
\end{aligned}
$$

Next let us estimate $\beta_{2}(n, z)$ in (2.30B):

$$
\begin{aligned}
&\left(1+\frac{n}{2}\right)^{N+1} e^{\omega n}\left|\beta_{2}(n, z)\right| \\
&=\left(1+\frac{n}{2}\right)^{N+1} e^{\omega n}\left|\left\langle\mathcal{S}^{-n} \mathcal{J} \hat{V},\left(z-B_{n}^{I}\right)^{-1} W_{n}^{-1} \tilde{T}_{n} W_{n} \mathcal{S}^{n} a_{n}\right\rangle\right| \\
& \leq \frac{1}{4} \sum_{\substack{|k-n| \geq n \\
k \neq \pm n}}\left(1+\frac{|k-n|}{2}\right)^{N} e^{\frac{\omega}{2}|n-k|}|\hat{V}(n-k)| \frac{1}{|n+k|} \\
& \quad \times \frac{1}{\left(1+\frac{|n+k|}{2}\right)^{N}}\left|\left(\tilde{T}_{n} W_{n} \mathcal{S}^{n} a_{n}\right)(k)\right| \\
& \quad+\frac{1}{4} \sum_{\substack{|k-n|<n \\
k \neq \pm n}} \frac{e^{\frac{\omega}{2}|n-k|}|\hat{V}(n-k)|}{|n-k|}\left|\left(\tilde{T}_{n} W_{n} \mathcal{S}^{n} a_{n}\right)(k)\right| \\
& \leq \frac{1}{2}\|V\|\left\|_{N, \omega}||\left|\tilde{T}_{n}\right|||\right\| W_{n} \mathcal{S}^{n} a_{n} \| .
\end{aligned}
$$

By Lemma 2.6 and (2.23), $\left\||| \tilde{T}_{n}\right\| \mid \leq \frac{\|V\|_{N, \omega}}{5 n}$. Further, $\left\|\left|\left(I d_{n}-T_{n}\right)^{-1}\right|\right\| \leq \frac{5}{4}$ for $n \geq n_{2}$, and therefore, by (2.29),

$$
\left\|W_{n} \mathcal{S}^{n} a_{n}\right\|_{l^{2}(\mathbb{Z}(n))}=\left\|\mathcal{S}^{n} a_{n}\right\|_{l_{\mathcal{S}^{n} w}^{2}(\mathbb{Z}(n))} \leq \frac{5}{4}\|V\|_{N, \omega} .
$$

Combining the estimates above, we obtain

$$
\sup _{|z| \leq M}\left(1+\frac{n}{2}\right)^{N+1} e^{\omega n}\left|\beta_{2}(n, z)\right| \leq \frac{\|V\|_{N, \omega}^{3}}{8 n} .
$$

Therefore

$$
\begin{gathered}
\left(\sum_{n \geq n_{2}}\left(1+\frac{n}{2}\right)^{2(N+1)} e^{2 \omega n} \sup _{|z| \leq M}\left|\beta_{2}(n, z)\right|^{2}\right)^{1 / 2} \\
\leq \frac{\|V\|_{N, \omega}^{3}}{8}\left(\sum_{n \geq n_{2}} \frac{1}{n^{2}}\right)^{1 / 2} \leq \frac{1}{8}\|V\|_{N, \omega}^{3} .
\end{gathered}
$$

Combined with (2.31), (2.30A) and (2.30B), the estimate (2.27). 
(ii) Proof of (2.28): The derivative $\frac{d}{d z} \beta(n, z)$ is given by

$$
\begin{aligned}
\frac{d}{d z} \beta(n, z)= & \frac{d}{d z}\left(\left\langle\mathcal{S}^{-n} \mathcal{J} \hat{V},\left(z-B_{n}^{I}\right)^{-1} \mathcal{S}^{n} \hat{V}\right\rangle+\left\langle\mathcal{S}^{-n} \mathcal{J} \hat{V},\left(z-B_{n}^{I}\right)^{-1} T_{n} \mathcal{S}^{n} a_{n}\right\rangle\right) \\
= & -\left\langle\mathcal{S}^{-n} \mathcal{J} \hat{V},\left(z-B_{n}^{I}\right)^{-2} \mathcal{S}^{n} \hat{V}\right\rangle-\left\langle\mathcal{S}^{-n} \mathcal{J} \hat{V},\left(z-B_{n}^{I}\right)^{-2} T_{n} \mathcal{S}^{n} a_{n}\right\rangle \\
& -\left\langle\mathcal{S}^{-n} \mathcal{J} \hat{V},\left(z-B_{n}^{I}\right)^{-1}\left(I d_{n}-T_{n}\right)^{-1} T_{n}\left(z-B_{n}^{I}\right)^{-1} \mathcal{S}^{n} a_{n}\right\rangle
\end{aligned}
$$

where we used the fact that the derivative of

$$
T_{n} \mathcal{S}^{n} a_{n}=T_{n}\left(I d_{n}-T_{n}\right)^{-1} \mathcal{S}^{n} \hat{V}=\left(\left(I d_{n}-T_{n}\right)^{-1}-I d_{n}\right) \mathcal{S}^{n} \hat{V}
$$

is given by

$$
\frac{d}{d z} T_{n} \mathcal{S}^{n} a_{n}=-\left(I d_{n}-T_{n}\right)^{-1} T_{n}\left(z-B_{n}^{I}\right)^{-1} \mathcal{S}^{n} a_{n}
$$

The three terms on the right hand side of (2.32) are estimated separately. The first one is estimated similarly as in (i): Using (2.31), one obtains

$$
\begin{aligned}
& \left(\sum_{n \geq n_{2}} \sup _{|z| \leq M}\left(1+\frac{n}{2}\right)^{2 N+4} e^{2 \omega n}\left|\left\langle\mathcal{S}^{-n} \mathcal{J} \hat{V},\left(z-B_{n}^{I}\right)^{-2} \mathcal{S}^{n} \hat{V}\right\rangle\right|^{2}\right)^{1 / 2} \\
& \quad \leq \frac{1}{4}\|V\|_{N, \omega}^{2} .
\end{aligned}
$$

The second term on the right hand side of (2.32) is estimated similarly as in (i), and one obtains

$$
\begin{aligned}
& \left(\sum_{n \geq n_{2}} \sup _{|z| \leq M}\left(1+\frac{n}{2}\right)^{2 N+4} e^{2 \omega n}\left|\left\langle\mathcal{S}^{-n} \mathcal{J} \hat{V},\left(z-B_{n}^{I}\right)^{-2} T_{n} \mathcal{S}^{n} a_{n}\right\rangle\right|^{2}\right)^{1 / 2} \\
& \quad \leq \frac{1}{8}\|V\|_{N, \omega}^{3} .
\end{aligned}
$$

To estimate the last term on the right hand side of (2.32), we first notice that

$$
\left(I d_{n}-T_{n}\right)^{-1} T_{n}=W_{n}^{-1}\left(I d_{n}-\tilde{T}_{n}\right)^{-1} \tilde{T}_{n} W_{n} .
$$

Thus, with $\tilde{S}_{n}:=\left(I d_{n}-\tilde{T}_{n}\right)^{-1} \tilde{T}_{n}$,

$$
\begin{aligned}
(1+ & \left.\frac{n}{2}\right)^{N+2} e^{\omega n}\left|\left\langle\mathcal{S}^{-n} \mathcal{J} \hat{V},\left(z-B_{n}^{I}\right)^{-1}\left(I d_{n}-T_{n}\right)^{-1} T_{n}\left(z-B_{n}^{I}\right)^{-1} \mathcal{S}^{n} a_{n}\right\rangle\right| \\
\leq & \frac{1}{4} \sum_{\substack{|k-n| \geq n \\
k \neq \pm n}}\left(1+\frac{|k-n|}{2}\right)^{N} e^{\frac{\omega}{2}|n-k|}|\hat{V}(n-k)| \\
& \times\left|\left(\tilde{S}_{n} W_{n}(1+n)\left(z-B_{n}^{I}\right)^{-1} \mathcal{S}^{n} a_{n}\right)(k)\right| \\
& +\frac{1}{4} \sum_{\substack{|k-n|<n \\
k \neq \pm n}} \frac{e^{\frac{\omega}{2}|n-k|}|\hat{V}(n-k)|}{|n-k|}\left|\left(\tilde{S}_{n} W_{n}(1+n)\left(z-B_{n}^{I}\right)^{-1} \mathcal{S}^{n} a_{n}\right)(k)\right| \\
\leq & \frac{1}{4}\|V\|_{N, \omega}\left\|\tilde{S}_{n} W_{n}(1+n)\left(z-B_{n}^{I}\right)^{-1} \mathcal{S}^{n} a_{n}\right\| \\
& +\frac{1}{4}\|V\|_{0, \omega}\left\|\tilde{S}_{n} W_{n}(1+n)\left(z-B_{n}^{I}\right)^{-1} \mathcal{S}^{n} a_{n}\right\| \\
\leq & \frac{1}{2}\|V\|_{N, \omega}\left\||| \tilde{T}_{n}\right\|\left|\left\|||\left(I d_{n}-\tilde{T}_{n}\right)^{-1} \mid\right\|\left\|W_{n}(1+n)\left(z-B_{n}^{I}\right)^{-1} \mathcal{S}^{n} a_{n}\right\| .\right.
\end{aligned}
$$


By Lemma 2.6 and (2.23), $\left\|\left|\tilde{T}_{n}\right|\right\| \leq \frac{\|V\|_{N, \omega}}{5 n}$ and $\left\|\left|\left(I d_{n}-\tilde{T}_{n}\right)^{-1}\right|\right\| \leq \frac{5}{4}$.

Further, for $n \geq n_{2}$

$$
\|\left.\left|(1+n)\left(z-B_{n}^{I}\right)^{-1}\right|\right|_{\left.\mathcal{L}_{\left(l_{\mathcal{S}^{n} w}^{2}\right.}(\mathbb{Z}(n))\right)} \leq \sup _{\substack{|z| \leq M \\ k \neq \pm n}}\left|\frac{1+n}{z-\pi^{2}(k-n)(k+n)}\right| \leq \frac{1}{4} .
$$

Thus, with (2.29),

$$
\left\|W_{n}(1+n)\left(z-B_{n}^{I}\right)^{-1} \mathcal{S}^{n} a_{n}\right\|_{l^{2}(\mathbb{Z}(n))} \leq \frac{1}{4} \cdot \frac{5}{4}\|V\|_{N, \omega} .
$$

Combining these estimates leads to

$$
\begin{aligned}
& \left(\sum_{n \geq n_{2}} \sup _{|z| \leq M}\left(1+\frac{n}{2}\right)^{2 N+4}\right. \\
& \left.\quad \times e^{2 \omega n}\left|\left\langle\mathcal{S}^{-n} \mathcal{J} \hat{V},\left(z-B_{n}^{I}\right)^{-1}\left(I d_{n}-T_{n}\right)^{-1} T_{n}\left(z-B_{n}^{I}\right)^{-1} \mathcal{S}^{n} a_{n}\right\rangle\right|^{2}\right)^{1 / 2} \\
& \quad \leq \frac{1}{32}\|V\|_{N, \omega}^{3}\left(\sum_{n \geq n_{2}} \frac{1}{n^{2}}\right)^{1 / 2} \leq \frac{1}{32}\|V\|_{N, \omega}^{3} .
\end{aligned}
$$

From (2.32) and (2.32i)-(2.32iii) the estimate (2.28) follows.

We are now ready to investigate (2.19).

Let

$$
r_{n}:=\max (|\hat{V}( \pm 2 n)|)+\max _{|z| \leq M}|\beta( \pm n, z)| .
$$

Notice that, by Proposition 2.7, for $n \geq n_{2}$,

$$
r_{n} \leq\|V\|+\|V\|_{N, \omega}^{2}\left(1+\frac{1}{8}\|V\|_{N, \omega}\right) .
$$

Proposition 2.8. Assume that $M \geq 10$ satisfies

$$
\|V\|+\|V\|_{N, \omega}^{2}\left(1+\frac{1}{8}\|V\|_{N, \omega}\right) \leq \frac{M}{4} .
$$

Then, for $n \geq n_{2}$, equation (2.19) has exactly two (counted with multiplicity) solutions $\zeta_{n}^{+}, \zeta_{n}^{-}$in $\overline{\mathcal{D}_{r_{n}}}$.

Proof. The result follows from Rouché's theorem. Clearly $\zeta^{2}=0$ has two roots in $\mathcal{D}_{r_{n}}$. For $|\zeta|=K r_{n}$ with $1<K<2$,

$$
\sup _{|z| \leq M}|(\hat{V}(2 n)+\beta(n, z))(\hat{V}(-2 n)+\beta(-n, z))| \leq r_{n}^{2}<\left|\zeta^{2}\right| .
$$

As $\beta\left( \pm n, z_{n}(\zeta)\right)$ depend analytically on $\zeta$ for $|\zeta|<\frac{M}{2}$ and $K r_{n}<2 r_{n} \leq \frac{M}{2}$, we deduce from Rouché's theorem that equation (2.19) has precisely two roots in $\mathcal{D}_{K r_{n}}$. As the two roots are independent of $K$ and $1<K<2$ is arbitrary close to 1 , we conclude that $\zeta_{n}^{ \pm} \in \overline{\mathcal{D}_{r_{n}}}$.

Let $z_{n}^{ \pm}=z\left(\zeta_{n}^{ \pm}\right)=\zeta_{n}^{ \pm}+\alpha\left(n, z_{n}^{ \pm}\right)$, where $\zeta_{n}^{ \pm}$are given by Proposition 2.8. Then

$$
\left|z_{n}^{+}-z_{n}^{-}\right| \leq\left|\zeta_{n}^{+}-\zeta_{n}^{-}\right|+\left(\sup _{|z| \leq M}\left|\frac{d}{d z} \alpha(n, z)\right|\right)\left|z_{n}^{+}-z_{n}^{-}\right| .
$$


By Lemma 2.4 (ii), (2.20) and as $n_{2} \geq n_{1}$,

$$
\sup _{|z| \leq M}\left|\frac{d}{d z} \alpha(n, z)\right| \leq \frac{1}{2}
$$

Together with $\left|\zeta_{n}^{+}-\zeta_{n}^{-}\right| \leq\left|\zeta_{n}^{+}\right|+\left|\zeta_{n}^{-}\right| \leq 2 r_{n}$, estimate (2.35) leads to

$$
\left|z_{n}^{+}-z_{n}^{-}\right| \leq 4 r_{n} \text {. }
$$

In view of the definition (2.33) of $r_{n}$, Proposition 2.7 and $\left|z_{n}^{+}-z_{n}^{-}\right|=\left|\lambda_{n}^{+}-\lambda_{n}^{-}\right|$, we conclude that

$$
\begin{aligned}
& \left(\sum_{n \geq n_{2}}\left(1+\frac{n}{2}\right)^{2 N} e^{2 \omega n}\left|\lambda_{n}^{+}-\lambda_{n}^{-}\right|^{2}\right)^{1 / 2} \\
& \quad \leq 8\left(\|V\|_{N, \omega}+\|V\|_{N, \omega}^{2}\left(1+\frac{1}{8}\|V\|_{N, \omega}\right)\right)<\infty .
\end{aligned}
$$

Next we want to obtain asymptotics for $\lambda_{n}^{+}-\lambda_{n}^{-}$. Rewrite (2.19) in two ways:

$$
\left(\zeta_{n}^{ \pm}\right)^{2}-\delta(n) \delta(-n)-\eta_{n}^{ \pm}=0,
$$

with $\eta_{n}^{ \pm}=\eta\left(z_{n}^{ \pm}\right)$, where $\delta(n), \eta(z)$ are defined either by (alternative 1 )

$$
\begin{aligned}
\delta(n) \equiv \delta^{I}(n):= & \hat{V}(2 n)+\beta(n, 0), \\
\eta(z) \equiv \eta^{I}(z):= & (\beta(-n, z)-\beta(-n, 0)) \hat{V}(2 n) \\
& +(\beta(n, z)-\beta(n, 0)) \hat{V}(-2 n) \\
& +\beta(-n, z) \beta(n, z)-\beta(-n, 0) \beta(n, 0)
\end{aligned}
$$

or by (alternative 2)

$$
\begin{aligned}
& \delta(n) \equiv \delta^{I I}(n):=\hat{V}(2 n), \\
& \eta(z) \equiv \eta^{I I}(z):=\beta(-n, z) \hat{V}(2 n)+\beta(n, z) \hat{V}(-2 n)+\beta(-n, z) \beta(n, z) .
\end{aligned}
$$

Introduce $s_{n}$, given by (alternative 1 )

$$
\begin{gathered}
s_{n} \equiv s_{n}^{I}:=\sup _{|z| \leq M}(|\beta(-n, z)-\beta(-n, 0)||\hat{V}(2 n)|+|\beta(n, z)-\beta(n, 0)||\hat{V}(-2 n)| \\
+|\beta(-n, z)||\beta(n, z)|+|\beta(-n, 0)||\beta(n, 0)|)
\end{gathered}
$$

or by (alternative 2 )

$$
s_{n} \equiv s_{n}^{I I}:=\sup _{|z| \leq M}(|\beta(-n, z)||\hat{V}(2 n)|+|\beta(n, z)||\hat{V}(-2 n)|+|\beta(-n, z)||\beta(n, z)|) .
$$

Use $|\beta(n, z)-\beta(n, 0)| \leq M \sup _{|z| \leq M}\left|\frac{d}{d z} \beta(n, z)\right|$ and Proposition 2.7 to obtain

$$
\begin{aligned}
& \sum_{n \geq n_{2}}\left(1+\frac{n}{2}\right)^{2 N+2} e^{2 \omega n} s_{n}^{I} \\
& \quad \leq \frac{M}{2}\|V\|_{N, \omega}^{3}\left(1+\|V\|_{N, \omega}\right)+2\|V\|_{N, \omega}^{4}\left(1+\frac{1}{8}\|V\|_{N, \omega}\right)^{2}
\end{aligned}
$$

and

$$
\begin{aligned}
& \sum_{n \geq n_{2}}\left(1+\frac{n}{2}\right)^{2 N+1} e^{2 \omega n} s_{n}^{I I} \\
& \quad \leq 2\|V\|_{N, \omega}^{3}\left(1+\frac{1}{8}\|V\|_{N, \omega}\right)+\|V\|_{N, \omega}^{4}\left(1+\frac{1}{8}\|V\|_{N, \omega}\right)^{2} .
\end{aligned}
$$


Proposition 2.9. Assume that $M \geq 10$ satisfies

$$
\|V\|+\|V\|_{N, \omega}^{2}\left(1+\frac{1}{8}\|V\|_{N, \omega}\right) \leq \frac{M}{4} .
$$

Then for $n \geq n_{2}$ and $\delta(n), s_{n}$ given by either $\delta^{I}(n), s_{n}^{I}$ or $\delta^{I I}(n), s_{n}^{I I}$, the roots $\zeta_{n}^{+}, \zeta_{n}^{-}$can be labeled in such a way that

(i) $\left.\mid \zeta_{n}^{+}-(\delta(n) \delta(-n))^{1 / 2}\right) \mid \leq 5 s_{n}^{1 / 2}$,

(ii) $\left|\zeta_{n}^{-}+(\delta(n) \delta(-n))^{1 / 2}\right| \leq 5 s_{n}^{1 / 2}$.

Proof. We consider two different cases:

Case 1. $|\delta(n) \delta(-n)| \leq 4 s_{n}$. This is an easy case for which (i) and (ii) are proved in the same way. Let us concentrate on (i). Then

$$
\begin{aligned}
\mid\left(\zeta_{n}^{+}\right. & \left.-(\delta(n) \delta(-n))^{1 / 2}\right)^{2}|\leq 2|\left(\zeta_{n}^{+}\right)^{2}|+2| \delta(n) \delta(-n) \mid \\
& \leq 2\left|\delta(n) \delta(-n)+\eta_{n}^{+}\right|+2|\delta(n) \delta(-n)| \\
& \leq 4|\delta(n) \delta(-n)|+2\left|\eta_{n}^{+}\right| \leq 18 s_{n} \leq\left(5 s_{n}^{1 / 2}\right)^{2},
\end{aligned}
$$

where for the second inequality, we used (2.38).

Thus $\left|\zeta_{n}^{+}-(\delta(n) \delta(-n))^{1 / 2}\right| \leq 5 s_{n}^{1 / 2}$.

Case 2. $|\delta(n) \delta(-n)| \geq 4 s_{n}$. Without any loss of generality we may assume that $s_{n}>0$. In particular, $|\delta(n) \delta(-n)|>0$. The equation (2.38) can than be rewritten as

$$
\left(\zeta_{n}\right)^{2}=\delta(-n) \delta(n)\left(1+\frac{\eta\left(z\left(\zeta_{n}\right)\right)}{\delta(-n) \delta(n)}\right)
$$

where $z\left(\zeta_{n}\right)$ is given by $(2.18)$. With

$$
\xi:=\frac{\zeta_{n}}{(\delta(-n) \delta(n))^{1 / 2}}
$$

formula (2.40) leads to

$$
(\xi)^{2}=\left(1+\frac{\eta\left(z\left(\zeta_{n}\right)\right)}{(\delta(-n) \delta(n))}\right) .
$$

As $|\delta(n) \delta(-n)| \geq 4 s_{n}$, one concludes that $\left|\frac{\eta\left(z\left(\zeta_{n}\right)\right)}{\delta(-n) \delta(n)}\right| \leq \frac{1}{4}$.

Denoting by $(1+w)^{1 / 2}$ the branch of the square root determined by $(1)^{1 / 2}=+1$, we obtain the equations

$$
\xi= \pm F(\xi)= \pm\left(1+\frac{\eta(z)}{\delta(n) \delta(-n)}\right)^{1 / 2}
$$

with $z=z\left(\zeta_{n}\right)=z\left((\delta(n) \delta(-n))^{1 / 2} \cdot \xi\right)$. Let us first consider $\left(2.42^{+}\right)$: Introduce $\mathcal{D}_{1 / 4}(1):=\left\{\xi \in \mathbb{C}|| \xi-1 \mid<\frac{1}{4}\right\}$ and notice that for $\xi \in \mathcal{D}_{1 / 4}(1), \zeta_{n}:=$ $(\hat{V}(2 n) \hat{V}(-2 n))^{1 / 2} \xi$ satisfies $\left|\zeta_{n}\right| \leq \frac{M}{4} \cdot \frac{5}{4}<\frac{M}{2}$.

As $\left|(1+x)^{1 / 2}-1\right| \leq|x|$ for $x \in \mathcal{D}_{1 / 4}(0)$ and case 2 holds, we conclude that $F$ maps $\overline{\mathcal{D}_{1 / 4}(1)}$ into itself. Moreover $F$ is continuous and therefore, according to Brower's fixed point theorem, admits at least one fixed point, denoted by $\xi^{I}$, i.e.

$$
\xi^{I}=+F\left(\xi^{I}\right)=+\left(1+\frac{\eta\left(z^{I}\right)}{\delta(n) \delta(-n)}\right)^{1 / 2}
$$


where $z^{I}=z\left((\hat{V}(-2 n) \hat{V}(2 n))^{1 / 2} \xi^{I}\right)$. Then, as we are in case 2 ,

$$
\begin{aligned}
\left|\xi^{I}-1\right| & \leq\left|\left(1+\frac{\eta\left(z^{I}\right)}{\delta(n) \delta(-n)}\right)^{1 / 2}-1\right| \leq \frac{\left|\eta\left(z^{I}\right)\right|}{|\delta(n) \delta(-n)|} \\
& \leq \frac{1}{2} \frac{s_{n}^{1 / 2}}{|\delta(n) \delta(-n)|^{1 / 2}}
\end{aligned}
$$

and, with $\zeta^{I}:=(\delta(n) \delta(-n))^{1 / 2} \xi^{I}$

$$
\left|\zeta^{I}-(\delta(-n) \delta(n))^{1 / 2}\right| \leq \frac{1}{2} s_{n}^{1 / 2}
$$

The same arguments can be used to show that there exists a solution $\xi^{I I} \in$ $\mathcal{D}_{1 / 4}(-1)$ of $\left(2.42^{-}\right)$so that, with $\zeta^{I I}:=(\delta(n) \delta(-n))^{1 / 2} \xi^{I I}$,

$$
\left|\zeta^{I I}+(\delta(n) \delta(-n))^{1 / 2}\right| \leq \frac{1}{2} s_{n}^{1 / 2} .
$$

It remains to show that $\left\{\zeta^{I}, \zeta^{I I}\right\}=\left\{\zeta_{n}^{+}, \zeta_{n}^{-}\right\}$. First notice that $\zeta^{I} \neq \zeta^{I I}$. Otherwise, we obtain a contradiction, by combining (2.43), (2.44), $s_{n}>0$ and the inequality case 2 as follows: Assume $\zeta^{I}=\zeta^{I I}=\zeta^{*}$. Then

$$
\begin{aligned}
0<2\left(2 s_{n}^{1 / 2}\right) & \leq\left|2(\delta(-n) \delta(n))^{1 / 2}\right| \leq \mid\left(\delta(n) \delta(-n)^{1 / 2}-\zeta^{*}+(\delta(n) \delta(-n))^{1 / 2}+\zeta^{*} \mid\right. \\
& \leq \frac{1}{2} s_{n}^{1 / 2}+\frac{1}{2} s_{n}^{1 / 2}=s_{n}^{1 / 2},
\end{aligned}
$$

and $0<4 s_{n}^{1 / 2} \leq s_{n}^{1 / 2}$ gives the claimed contradiction.

Further notice that $\zeta^{I}, \zeta^{I I}, \zeta_{n}^{+}, \zeta_{n}^{-}$are all solutions of (2.40). But according to Proposition 2.8, equation (2.40) has precisely two solutions. Therefore $\left\{\zeta^{I}, \zeta^{I I}\right\}=$ $\left\{\zeta_{n}^{+}, \zeta_{n}^{-}\right\}$. This proves Proposition 2.9 in case 2 .

We are now ready to prove Theorem 2 . It is contained in the following

Theorem 2.10. Let $S^{1}=\mathbb{R} / \mathbb{Z}, N \in \mathbb{Z}_{\geq 0}, \omega \in \mathbb{R}_{\geq 0}$ and $M \geq 10$.

Then, for any $V \in H^{N, \omega}\left(S^{1} ; \mathbb{C}\right)$ with $\|V\|+\|V\|_{N, \omega}^{2}\left(1+\frac{1}{8}\|V\|_{N, \omega}\right) \leq \frac{M}{4}$,

(i) $\left(\sum_{n \geq M^{2}}\left(1+\frac{n}{2}\right)^{2 N+1} e^{2 \omega n}\left|\left(\lambda_{n}^{+}-\lambda_{n}^{-}\right)-2(\hat{V}(-2 n) \hat{V}(2 n))^{1 / 2}\right|^{2}\right)^{1 / 2} \leq 3 M^{2}$,

$$
\begin{aligned}
& \left(\sum_{n \geq M^{2}}\left(1+\frac{n}{2}\right)^{2 N+2} e^{2 \omega n}\right. \\
& \left.\quad \times\left|\left(\lambda_{n}^{+}-\lambda_{n}^{-}\right)-2((\hat{V}(-2 n)+\beta(-n, 0))(\hat{V}(2 n)+\beta(n, 0)))^{1 / 2}\right|^{2}\right)^{1 / 2} \leq 5 M^{2},
\end{aligned}
$$

where, for $n \geq M^{2}, \lambda_{n}^{ \pm}=n^{2} \pi^{2}+z_{n}^{ \pm}$(and thus $\left\{\lambda_{n}^{+}, \lambda_{n}^{-}\right\}=\left\{\lambda_{2 n}, \lambda_{2 n-1}\right\}$ ) with $\left(\lambda_{n}\right)_{n \geq 0}$ denoting the periodic spectrum (ordered as explained in the introduction) of $-\frac{\bar{d}^{2}}{d x^{2}}+V$ considered on the interval $\left.[0,2]\right)$ and $(\hat{V}(k))_{k \in \mathbb{Z}}$ are the Fourier coefficients of $V$ when considered as functions with period 2.

Proof. Without any loss of generality we assume that $V \in H_{0}^{N, \omega}\left(S^{1} ; \mathbb{C}\right)$. Statements (i) and (ii) are proved in the same way, so we concentrate on (i). Notice that $M^{2} \geq n_{2}:=\max \left(\frac{M+1}{2},\|V\|^{2},\|V\|_{N, \omega}\right)$. 
For $n \geq M^{2}, \lambda_{n}^{+}-\lambda_{n}^{-}=z_{n}^{+}-z_{n}^{-}$. Furthermore, $z_{n}^{ \pm}=\alpha\left(n, z_{n}^{ \pm}\right)+\zeta_{n}^{ \pm}$, and, by Proposition 2.9,

$$
\left|\left(\zeta_{n}^{+}-\zeta_{n}^{-}\right)-2(\hat{V}(-2 n) \hat{V}(2 n))^{1 / 2}\right| \leq 10 s_{n}^{1 / 2} .
$$

Therefore

$$
\begin{aligned}
\mid \lambda_{n}^{+}- & \lambda_{n}^{-}-2(\hat{V}(-2 n) \hat{V}(2 n))^{1 / 2} \mid \\
& \leq\left|\zeta_{n}^{+}-\zeta_{n}^{-}-2(\hat{V}(-2 n) \hat{V}(2 n))^{1 / 2}\right|+\left(\sup _{|z| \leq M}\left|\frac{d}{d z} \alpha(n, z)\right|\right)\left|z_{n}^{+}-z_{n}^{-}\right| \\
& \leq 10 s_{n}^{1 / 2}+\frac{\|V\|^{2}}{n^{2}}\left|z_{n}^{+}-z_{n}^{-}\right|,
\end{aligned}
$$

where for the last inequality, we used Lemma 2.4 (ii) and (2.26). By (2.37)

$$
\begin{aligned}
& \left(\sum_{n \geq M^{2}}\left(1+\frac{n}{2}\right)^{2 N+4} e^{2 \omega n}\left(\frac{\|V\|^{2}}{n^{2}}\left|z_{n}^{+}-z_{n}^{-}\right|\right)^{2}\right)^{1 / 2} \\
& \quad \leq 2 \cdot\|V\|^{2} 8\left(\|V\|_{N, \omega}+\|V\|_{N, \omega}^{2}\left(1+\frac{1}{8}\|V\|_{N, \omega}\right)\right) \leq 2 M^{2} .
\end{aligned}
$$

By $\left(2.39^{\mathrm{II}}\right)$,

$$
10 \cdot\left(\sum_{n \geq M^{2}}\left(1+\frac{n}{2}\right)^{2 N+1} e^{2 \omega n} s_{n}\right)^{1 / 2} \leq 5 M
$$

As $M \geq 10,5 M \leq M^{2}$, and therefore from (2.45), (2.46) and (2.47) we obtain

$$
\left(\sum_{n \geq M^{2}}\left(1+\frac{n}{2}\right)^{2 N+1} e^{2 \omega n}\left|\lambda_{n}^{+}-\lambda_{n}^{-}-2(\hat{V}(-2 n) \hat{V}(2 n))^{1 / 2}\right|^{2}\right)^{1 / 2} \leq 3 M^{2}
$$

Remark 1 . Theorem 2.10 can be improved for real valued potentials. It leads to a result obtained by Marčenko [Ma]:

Theorem 2.10 A. Let $N \in \mathbb{Z}_{\geq 0}$ and $M \geq 10$. Then, for any $V \in H^{N, \omega}\left(S^{1} ; \mathbb{R}\right)$ with $\|V\|+\|V\|_{N, \omega}^{2}\left(1+\frac{1}{8}\|V\|_{N, \omega}\right) \leq \frac{M}{4}$,

$$
\left.\left.\sum_{n \geq M^{2}}\left(1+\frac{n}{2}\right)^{2 N+2} e^{2 \omega n}\left|\left(\lambda_{n}^{+}-\lambda_{n}^{-}\right)-2\right| \hat{V}(-2 n) \hat{V}(2 n)\right|^{1 / 2}\right|^{2} \leq 3 M^{2}
$$

Remark for Theorem 2.10 A. Recall that

$$
\left(\zeta_{n}\right)^{2}-\hat{V}(2 n) \hat{V}(-2 n)-\eta_{n}=0
$$

If $V$ is real valued, then $|\hat{V}(2 n)|=|\hat{V}(-2 n)|$. This is a quantitative version of the following statement:

$$
\hat{V}(2 n) \cdot \hat{V}(-2 n)=0 \Longleftrightarrow \hat{V}(2 n)=0 \text { and } \hat{V}(-2 n)=0 .
$$

To improve on Theorem 2.10 as in Theorem 2.10 A, it seems that one needs to restrict to potentials satisfying a vanishing condition which is a quantitative version of $(2.50)$. 
Proof of Theorem 2.10 A. Without any loss of generality, $V \in H_{0}^{N, \omega}\left(S^{1} ; \mathbb{R}\right)$, i.e. $\hat{V}(0)=0$. If $V$ is real valued, then $\hat{V}(-k)=\overline{\hat{V}(k)}$, and

$$
\begin{aligned}
& \beta(-n, z)=\overline{\beta(n, \bar{z})} \quad\left(|z| \leq M, n \geq n_{2}\right), \\
& \overline{\alpha(n, z)}=\alpha(n, \bar{z}) \quad\left(|z| \leq M, n \geq n_{2}\right) .
\end{aligned}
$$

Further, $-\frac{d^{2}}{d x^{2}}+V$ is selfadjoint, and therefore, the perodic spectrum of $-\frac{d^{2}}{d x^{2}}+V$ is contained in $\mathbb{R}$. Following the proof of Theorem 2.10, we know that for $n \geq 2 M^{2}$ we have $\lambda_{n}^{ \pm}=n^{2} \pi^{2}+z_{n}^{ \pm}$, and thus $z_{n}^{ \pm} \in \mathbb{R}$. Moreover, $\zeta_{n}^{ \pm}=z_{n}^{ \pm}-\alpha\left(n, z_{n}^{ \pm}\right) \in \mathbb{R}$, as $\alpha\left(n, z_{n}^{ \pm}\right) \in \mathbb{R}$ by $(2.52)$.

Therefore, equation (2.19) can be written as

$$
\left(\zeta_{n}^{ \pm}\right)^{2}=\left|\hat{V}(2 n)+\beta\left(n, z\left(\zeta_{n}^{ \pm}\right)\right)\right|^{2},
$$

which leads to

$$
\zeta_{n}^{ \pm}= \pm\left|\hat{V}(2 n)+\beta\left(n, z\left(\zeta_{n}^{ \pm}\right)\right)\right|
$$

Thus

$$
\begin{aligned}
\left|\zeta_{n}^{ \pm}-( \pm|\hat{V}(2 n)|)\right| & \leq|| \hat{V}(2 n)+\beta\left(n, z\left(\zeta_{n}^{ \pm}\right)\right)|-| \hat{V}(2 n)|| \\
& \leq\left|\beta\left(n, z\left(\zeta_{n}^{ \pm}\right)\right)\right|
\end{aligned}
$$

and, by Proposition 2.7,

$$
\begin{aligned}
& \left(\sum_{n \geq n_{2}}\left(1+\frac{n}{2}\right)^{2 N+2} e^{2 \omega n}\left|\left(\zeta_{n}^{+}-\zeta_{n}^{-}\right)-2\right| \hat{V}(2 n)||\right)^{1 / 2} \\
& \leq\|V\|_{N, \omega}^{2}\left(1+\frac{1}{8}\|V\|_{N, \omega}\right) \leq \frac{M}{4}
\end{aligned}
$$

Combining with (2.45) and (2.46), one obtains

$$
\begin{aligned}
& \left(\sum_{n \geq M^{2}}\left(1+\frac{n}{2}\right)^{2 N+2} e^{2 \omega n}\left|\left(\lambda_{n}^{+}-\lambda_{n}^{-}\right)-2\right| \hat{V}(2 n)||^{2}\right)^{1 / 2} \\
& \quad \leq 2 M^{2}+\frac{M}{4} \leq 3 M^{2} .
\end{aligned}
$$

Remark 2. If $V$ is an even, possibly complex valued potential, Theorem 2.10 can be reformulated in a way which leads to an improvement. In the case when $V \in H^{N, \omega}\left(S^{1} ; \mathbb{C}\right)$ is even, i.e. $V(x)=V(-x)$, it is a well known fact that for $n$ sufficiently large, $\left\{\lambda_{n}^{+}, \lambda_{n}^{-}\right\}=\left\{\mu_{n}, \nu_{n}\right\}$, where $\left(\mu_{n}\right)_{n \geq 1}$ denote the Dirichlet eigenvalues and $\left(\nu_{n}\right)_{n \geq 0}$ denote the Neumann eigenvalues of $-\frac{d^{2}}{d x^{2}}+V$ considered on the unit interval. Further, the eigenfunctions of the Dirichlet eigenvalues are odd, whereas the eigenfunctions of the Neumann eigenvalues are even.

Theorem 2.10 B. Let $N \in \mathbb{Z}_{\geq 0}, \omega \in \mathbb{R}_{\geq 0}$ and $M \geq 10$.

Then, for any even potential $V \in H^{N, \omega}\left(S^{1}, \mathbb{C}\right)$ satisfying

$$
\|V\|+\|V\|_{N, \omega}^{2}\left(1+\frac{1}{8}\|V\|_{N, \omega}\right) \leq \frac{M}{4},
$$

we have

$$
\left(\sum_{n \geq M^{2}}\left(1+\frac{n}{2}\right)^{2 n+2} e^{2 \omega n}\left|\left(\mu_{n}-\nu_{n}\right)+2 \hat{V}(2 n)\right|^{2}\right)^{1 / 2} \leq 3 M^{2} .
$$


Proof. In the case where $V$ is even one verifies that

$$
\hat{V}(2 k)=\hat{V}(-2 k) ; \beta(-k, z)=\beta(k, z) .
$$

Therefore, equation (2.19) leads to $\zeta_{n}^{2}=\left(\hat{V}(2 n)+\beta\left(n, z\left(\zeta_{n}\right)\right)\right)^{2}$ and, in turn,

$$
\zeta_{n}^{ \pm}= \pm\left(\hat{V}(2 n)+\beta\left(n, z\left(\zeta_{n}^{ \pm}\right)\right)\right) .
$$

Then, with $\varepsilon_{n}= \pm$,

$$
\mu_{n}=n^{2} \pi^{2}+\alpha\left(n, z\left(\zeta_{n}^{\varepsilon_{n}}\right)\right)+\varepsilon_{n}\left(\hat{V}(2 n)+\beta\left(n, z\left(\zeta_{n}^{\varepsilon_{n}}\right)\right)\right)
$$

and

$$
\nu_{n}=n^{2} \pi^{2}+\alpha\left(n, z\left(\zeta_{n}^{-\varepsilon_{n}}\right)\right)-\varepsilon_{n}\left(\hat{V}(2 n)+\beta\left(n, z\left(\zeta_{n}^{-\varepsilon_{n}}\right)\right)\right) .
$$

To determine the sign $\varepsilon_{n}$, recall that the eigenfunction $y_{2}\left(x, \mu_{n}\right)$ is odd. Its Fourier coefficients $(a(k ; n))_{k \in \mathbb{Z}}$ therefore satisfy $a(-k ; n)=-a(k ; n)$.

Thus, in equation (2.14), $x=-y$. Together with

$$
-z\left(\zeta^{\varepsilon_{n}}\right)+\alpha\left(n, z\left(\zeta^{\varepsilon_{n}}\right)\right)=\zeta^{\varepsilon_{n}}=\varepsilon_{n}\left(\hat{V}(2 n)+\beta\left(n, z\left(\zeta_{n}^{\varepsilon_{n}}\right)\right)\right),
$$

equation (2.14) implies

$$
\left(\varepsilon_{n}\left(\hat{V}(2 n)+\beta\left(n, z\left(\zeta^{\varepsilon_{n}}\right)\right)\right)+\hat{V}(2 n)+\beta\left(n, z\left(\zeta^{\varepsilon_{n}}\right)\right)\right) x=0 .
$$

Since, in view of $(2.5)-(2.7),(x, y) \neq(0,0)$ (for $\left.n \geq n_{0}\right)$ (otherwise, $a(k, n)=0$ for all $k$ ) we then conclude that $\varepsilon_{n}=-1$ as claimed. As a consequence,

$$
\mu_{n}-\nu_{n}=-2 \hat{V}(2 n)-\beta\left(n, z_{n}^{+}\right)-\beta\left(n, z_{n}^{-}\right)+\alpha\left(n, z_{n}^{-}\right)-\alpha\left(n, z_{n}^{+}\right) .
$$

As in the proof of Theorem 2.10 A, one then obtains, by Proposition 2.7, combined with (2.45) and (2.46),

$$
\left(\sum_{n \geq 2 M^{2}}\left(1+\frac{n}{2}\right)^{2 N+2} e^{2 \omega n}\left|\mu_{n}-\nu_{n}+2 \hat{V}(2 n)\right|^{2}\right)^{1 / 2} \leq 3 M^{2} .
$$

As an application of Theorem 2.10 we obtain asymptotic estimates of the eigenvalues $\lambda_{n}^{ \pm}$

$$
\begin{aligned}
\lambda_{n}^{ \pm}= & n^{2} \pi^{2}+\alpha\left(n, \frac{z_{n}^{+}+z_{n}^{-}}{2}\right) \\
& \pm((\hat{V}(-2 n)+\beta(-n, 0))(\hat{V}(2 n)+\beta(n, 0)))^{1 / 2}+l_{N+1, \omega}^{2}(n),
\end{aligned}
$$

and of $\tau_{n}:=\frac{\lambda_{n}^{+}+\lambda_{n}^{-}}{2}$

$$
\tau_{n}=n^{2} \pi^{2}+\alpha\left(n, \frac{z_{n}^{+}+z_{n}^{-}}{2}\right)+l_{N+1, \omega}^{2}(n),
$$

where, by abuse of notation, we mean by $\left(l_{N+1, \omega}^{2}(n)\right)_{n \geq 1}$ an element in $l_{N+1, \omega}^{2}(\mathbb{N})$.

We finish this section with a brief discussion of the linear space $E_{n}$ spanned by eigenfunctions $f_{n}^{+}, f_{n}^{-}$corresponding to simple eigenvalues $\lambda_{n}^{+} \neq \lambda_{n}^{-}$, and of the root space $E_{n}$ corresponding to double eigenvalues $\lambda_{n}^{+}=\lambda_{n}^{-}$.

For a function $f(x)=\sum_{k \in \mathbb{Z}} \hat{f}(k) e^{i k \pi x}$ in $E_{n}$, the part $\sum_{k \neq \pm n} \hat{f}(k) e^{i k \pi x}$ is small when compared with $\hat{f}(n) e^{i n \pi x}+\hat{f}(-n) e^{-i n \pi x}$. This follows from the following result, which we will use in section 3 .

In view of $(2.7)$, we introduce $a_{x, y, z} \in l^{2}(\mathbb{Z}(n))$ :

$$
a_{x, y, z}:=x\left(z-B_{n}\right)^{-1}\left(\mathcal{S}^{n} \hat{V}\right)_{\mathbb{Z}(n)}+y\left(z-B_{n}\right)^{-1}\left(\mathcal{S}^{-n} \hat{V}\right)_{\mathbb{Z}(n)} .
$$


Proposition 2.11. Assume $V \in H_{0}^{N, \omega}\left(S^{1} ; \mathbb{C}\right)$. Then for $|z| \leq M, n \geq n_{2}$ and $x, y \in \mathbb{C}$

(i) $\left\|\left(z-B_{n}\right)^{-1}\left(\mathcal{S}^{n} V\right)_{\mathbb{Z}(n)}\right\|_{l_{\mathcal{S}^{n} w}^{2}(\mathbb{Z}(n))} \leq \frac{1}{n}\|V\|_{N, \omega}$;

(ii) $\left\|\left(z-B_{n}\right)^{-1}\left(\mathcal{S}^{-n} V\right)_{\mathbb{Z}(n)}\right\|_{l_{\mathcal{S}^{-n} n_{w}}^{2}(\mathbb{Z}(n))} \leq \frac{1}{n}\|V\|_{N, \omega}$;

(iii) $\left(\sum_{k \neq \pm n} e^{|| k|-n| \omega}\left(1+\left|\frac{|k|-n}{2}\right|\right)^{2 N}\left|a_{x, y, z}(k)\right|^{2}\right)^{1 / 2} \leq \frac{|x|+|y|}{n}\|V\|_{N, \omega}$;

(iv) $\left\|\left(z-B_{n}\right)^{-1}\left(\mathcal{S}^{n} V\right)_{\mathbb{Z}(n)}\right\|_{l^{1}(\mathbb{Z}(n))} \leq \frac{1}{n}\|V\|$;

(v) $\left\|\left(z-B_{n}\right)^{-1}\left(\mathcal{S}^{-n} V\right)_{\mathbb{Z}(n)}\right\|_{l^{1}(\mathbb{Z}(n))} \leq \frac{1}{n}\|V\|$;

(vi) $\sum_{k \neq \pm n}\left|a_{x, y, z}(k)\right| \leq \frac{|x|+|y|}{n}\|V\|$.

Remark. Notice that, with $w_{1}:=\mathcal{S}^{n} w, w_{2}:=\mathcal{S}^{-n} w$, the function

$$
w_{1} \wedge w_{2}(k):=\min \left(w_{1}(k), w_{2}(k)\right)
$$

is given by

$$
\begin{aligned}
w_{1} \wedge w_{2}(k) & =\left(1+\left|\frac{|k|-n}{2}\right|\right)^{N} e^{\frac{\omega}{2}|| k|-n|} \\
& = \begin{cases}w_{1}(k) & \text { for } \quad k \leq 0, \\
w_{2}(k) & \text { for } k \geq 0\end{cases}
\end{aligned}
$$

Furthermore,

$$
\sup _{k} \frac{w_{1}(k)}{w_{2}(k)} \leq(1+n)^{N} e^{n \omega} \quad \text { and } \quad \sup _{k} \frac{w_{2}(k)}{w_{1}(k)} \leq(1+n)^{N} e^{n \omega} .
$$

This implies that $w_{1} \wedge w_{2}$ is a weight with $M_{w_{1} \wedge w_{2}}=\left((1+n)^{N} e^{n \omega}\right)^{2}$. Thus $M_{w_{1} \wedge w_{2}}$ is increasing in $n$.

Proof. (i) By Lemma 2.6,

$$
\left\|T_{n} \mid\right\|_{\mathcal{L}\left(l_{\mathcal{S}^{n} w}^{2}(\mathbb{Z}(n))\right)} \leq \frac{\|V\|_{N, \omega}}{2 n}
$$

and thus, for $|z| \leq M$ and $n \geq n_{2}$,

$$
\begin{gathered}
\left\|\left|\left(z-B_{n}\right)^{-1}\right|\right\|_{\left.\mathcal{L}_{\left(l_{\mathcal{S}^{n} w}^{2}\right.}(\mathbb{Z}(n))\right)}=\left|\left\|\left(z-B_{n}^{I}\right)^{-1} \cdot\left(I d_{n}-T_{n}\right)^{-1} \mid\right\|_{\mathcal{L}\left(l_{\mathcal{S}^{n} w}^{2}(\mathbb{Z}(n))\right)}\right. \\
\leq 2\left\|||\left(z-B_{n}^{I}\right)^{-1} \mid\right\|_{\mathcal{L}\left(l_{\mathcal{S}^{n} w}^{2}(\mathbb{Z}(n))\right)} \leq 2 \cdot \frac{1}{2 n},
\end{gathered}
$$

where for the last inequality we used Lemma 2.1. This implies that

$$
\left\|\left(z-B_{n}\right)^{-1}\left(\mathcal{S}^{n} V\right)_{\mathbb{Z}(n)}\right\|_{l_{\mathcal{S}^{n} w}^{2}(\mathbb{Z}(n))} \leq \frac{1}{n}\left\|\mathcal{S}^{n} V\right\|_{\mathcal{S}^{n} w} \leq \frac{1}{n}\|V\|_{N, \omega} .
$$

(ii) Using the same arguments as in the proof of Lemma 2.6, one shows that

$$
\left\||| T_{n} \mid\right\|_{\mathcal{L}\left(l_{\mathcal{S}^{-n} w^{2}}(\mathbb{Z}(n))\right)} \leq \frac{\|V\|_{N, \omega}}{2 n} .
$$

One then argues as in the proof of (i) to conclude (ii).

(iii) Notice that for $k \in \mathbb{Z}, n \geq 1$

$$
|| k|-n|=\min (|k-n|,|k+n|) .
$$

Thus

$$
\left(1+\left|\frac{|k|-n}{2}\right|\right)^{N} e^{\frac{\omega}{2}|| k|-n|} \leq \min \left(\left(\mathcal{S}^{n} w\right)(k),\left(\mathcal{S}^{-n} w\right)(k)\right)
$$

and (iii) follows from (i), (ii) and (2.56). 
(iv) We have

$$
\begin{aligned}
\|(z & \left.-B_{n}\right)^{-1}\left(\mathcal{S}^{n} V\right)_{\mathbb{Z}(n)}\left\|_{l^{1}(\mathbb{Z}(n))}=\right\|\left(z-B_{n}^{I}\right)^{-1}\left(I d_{n}-T_{n}\right)^{-1}\left(\mathcal{S}^{n} V\right)_{\mathbb{Z}(n)} \|_{l^{1}(\mathbb{Z}(n))} \\
& =\sum_{k \neq \pm n} \frac{1}{\left|z-\pi^{2}\left(k^{2}-n^{2}\right)\right|}\left|\left(I d_{n}-T_{n}\right)^{-1}\left(\mathcal{S}^{n} V\right)(k)\right| \\
& \leq\left(\sum_{k \neq \pm n} \frac{1}{\left|z-\pi^{2}\left(k^{2}-n^{2}\right)\right|^{2}}\right)^{1 / 2}\left\|\left(I d_{n}-T_{n}\right)^{-1}\left(\mathcal{S}^{n} V\right)_{\mathbb{Z}(n)}\right\| \\
& \leq \frac{1}{2 n} \cdot 2 \cdot\|V\|,
\end{aligned}
$$

where for the last inequality we used Lemma 2.1 (and its proof).

(v) is proved in the same way as (iv).

(vi) follows from (iv) and (v).

\section{Proof of Theorem 1}

To prove Theorem 1, we follow the same scheme used in [BKM1, section 2]. Recall that the map $\Lambda$ (cf. [BBGK]) is constructed in two steps. First let us consider the map $\Phi: L_{0}^{2}\left(S^{1}\right) \rightarrow l^{2}\left(\mathbb{R}^{2}\right)$, introduced and analyzed in [Ka]. For $V \in L_{0}^{2}\left(S^{1}\right)$, denote by $E_{n}$ the image of the Riesz projector $(n \geq 1)$

$$
P_{n}:=\frac{1}{2 \pi i} \int_{\Gamma_{n}}\left(z-\left(-\frac{d^{2}}{d x^{2}}+V\right)\right)^{-1} d z
$$

where $\Gamma_{n}$ is a counterclockwise oriented circle with center $\tau_{n}(V):=\left(\lambda_{n}^{+}+\lambda_{n}^{-}\right) / 2$ of radius bigger than $\frac{\gamma_{n}}{2}=\frac{\lambda_{n}^{+}-\lambda_{n}^{-}}{2}$, but sufficiently small so that all eigenvalues different from $\lambda_{n}^{+}, \lambda_{n}^{-}$are outside of $\Gamma_{n}$. Here, for convenience, we set $\lambda_{n}^{+} \equiv \lambda_{2 n}$ and $\lambda_{n}^{-} \equiv \lambda_{2 n-1}$.

We choose in $E_{n}$ a basis

$$
\begin{aligned}
& G_{2 n-1}(x) \equiv G_{n}^{-}(x)=\sum_{k \in \mathbb{Z}} \hat{G}_{n}^{-}(k) e^{i k \pi x}, \\
& G_{2 n}(x) \equiv G_{n}^{+}(x)=\sum_{k \in \mathbb{Z}} \hat{G}_{n}^{+}(k) e^{i k \pi x}
\end{aligned}
$$

normalized as follows (the normalization conditions are written in such a way that they remain unchanged if we consider small complex valued perturbations of $V$ ):

$$
\begin{gathered}
\sum_{k \in \mathbb{Z}} \hat{G}_{n}^{ \pm}(k) \hat{G}_{n}^{ \pm}(-k)=1, \\
0=G_{n}^{-}(0)=\sum_{k \in \mathbb{Z}} \hat{G}_{n}^{-}(k), \\
0=\left\langle G_{n}^{-}, G_{n}^{+}\right\rangle=\sum_{k \in \mathbb{Z}} \hat{G}_{n}^{-}(k) \hat{G}_{n}^{+}(-k) .
\end{gathered}
$$

The signs of $G_{n}^{-}$and $G_{n}^{+}$are determined in such a way that (with' $=\frac{d}{d x}$ )

$$
\operatorname{Re}\left(\left(G_{n}^{-}\right)^{\prime}(0)\right)>0
$$




$$
\operatorname{Re}\left(\operatorname{det}\left(\begin{array}{cc}
G_{n}^{+}(0) & G_{n}^{-}(0) \\
\left(G_{n}^{+}\right)^{\prime}(0) & \left(G_{n}^{-}\right)^{\prime}(0)
\end{array}\right)\right)>0 .
$$

The map $\Phi(V):=\left(\Phi_{n}(V)\right)_{n \geq 1}$ is then defined by

$$
\Phi_{n}(V):=\left(\begin{array}{c}
\int_{S^{1}} G_{n}^{+}(x)\left(-\frac{d^{2}}{d x^{2}}+V-\tau_{n}\right) G_{n}^{+}(x) d x \\
\int_{S^{1}} G_{n}^{-}(x)\left(-\frac{d^{2}}{d x^{2}}+V-\tau_{n}\right) G_{n}^{+}(x) d x
\end{array}\right) .
$$

According to [Ka] (cf. also [BBGK, section 4]), $\Phi: L_{0}^{2}\left(S^{1}\right) \rightarrow l^{2}\left(\mathbb{R}^{2}\right)$ is real analytic. Denote by $\Phi^{(N, \omega)}$ the restriction $\Phi^{(N, \omega)}:=\left.\Phi\right|_{H_{0}^{N, \omega}\left(S^{1}\right)}$. Note that Theorem 2 implies that $\Phi^{(N, \omega)}\left(H_{0}^{N, \omega}\left(S^{1}\right)\right) \subseteq l_{N, \omega}^{2}\left(\mathbb{R}^{2}\right)$.

Since $\Phi: L_{0}^{2}\left(S^{1}\right) \rightarrow l^{2}\left(\mathbb{R}^{2}\right)$ is real analytic, for any $V_{0} \in L_{0}^{2}\left(S^{1}\right)$, there exists a neighborhood $\mathcal{U}$ of $V_{0}$ in $L_{0}^{2}\left(S^{1} ; \mathbb{C}\right)$ so that $\Phi$ can be extended to an analytic map, $\Phi: \mathcal{U} \rightarrow l^{2}\left(\mathbb{N} ; \mathbb{C}^{2}\right)$. Thus $\Phi^{(N, \omega)}$ extends to a map on $\mathcal{U} \cap H_{0}^{N, \omega}\left(S^{1} ; \mathbb{C}\right)$.

Proposition 3.1. Assume $V_{0} \in H_{0}^{N, \omega}\left(S^{1} ; \mathbb{R}\right)\left(N \in \mathbb{Z}_{\geq 0}, \omega \in \mathbb{R}_{\geq 0}\right)$.

(i) Then there exist a neighborhood $\mathcal{U}$ of $V_{0}$ in $H_{0}^{N, \omega}\left(S^{1} ; \mathbb{C}\right)$ and $1 \leq C<\infty$ such that $\Phi_{n}$ is analytic on $\mathcal{U}(n \geq 1)$ and, for any $V \in \mathcal{U}$,

$$
\sum_{n \geq 1}(1+n)^{2 N+2} e^{2 n \omega}\left\|\Phi_{n}(V)-\left(\frac{\hat{V}(2 n)+\hat{V}(-2 n)}{2}\right)\right\|^{2} \leq C .
$$

(ii) $\Phi^{(N, \omega)}: H_{0}^{N, \omega}\left(S^{1}\right) \rightarrow l_{N, \omega}^{2}\left(\mathbb{R}^{2}\right)$ is real analytic.

Proof. (ii) From (i) we conclude that $\Phi^{(N, \omega)}(\mathcal{U})$ is a bounded subset of $l_{N, \omega}^{2}\left(\mathbb{N} ; \mathbb{C}^{2}\right)$. Moreover, $\Phi_{n}$ is analytic on $\mathcal{U}$ for any $n \geq 1$. As $V_{0} \in H_{0}^{N, \omega}\left(S^{1}\right)$ is arbitrary, this implies that $\Phi^{(N, \omega)}: H_{0}^{N, \omega}\left(S^{1}\right) \rightarrow l_{N, \omega}^{2}\left(\mathbb{R}^{2}\right)$ is real analytic (cf. [PT, Appendix A]).

(i) As $\Phi^{(N, \omega)}$ is the restriction of $\Phi$ and $\Phi$ is locally bounded, it suffices to find $\mathcal{U}, C, n_{3} \geq 1$ such that for $V \in \mathcal{U}$

$$
\sum_{n \geq n_{3}}(1+n)^{2 N+2} e^{2 n \omega}\left\|\Phi_{n}(V)-\left(\begin{array}{c}
(\hat{V}(2 n)+\hat{V}(-2 n)) / 2 \\
(\hat{V}(2 n)-\hat{V}(-2 n) / 2 i
\end{array}\right)\right\|^{2} \leq C .
$$

To prove (3.8) we consider the cases where $\lambda_{n}^{+}=\lambda_{n}^{-}$and $\lambda_{n}^{+} \neq \lambda_{n}^{-}$separately. The statement follows from Lemma 3.2 and Lemma 3.3 below.

Let us first treat the case where $\lambda_{n}^{+}=\lambda_{n}^{-}$is a double eigenvalue of $-\frac{d^{2}}{d x^{2}}+V$ for $V \in H_{0}^{N, \omega}\left(S^{1}, \mathbb{C}\right)$. Let $\mathcal{M}=\left\{n \geq 2 n_{2} \mid \lambda_{n}^{+}=\lambda_{n}^{-}\right\}$, where $n_{2}$ is given by (2.26). For $n \in \mathcal{M}$ we have $z_{n}^{+}=z_{n}^{-}$, and therefore

$$
\zeta_{n}^{+}=\zeta_{n}^{-} .
$$

Together with $\left(\zeta_{n}^{ \pm}\right)^{2}=A$, where $A=\left(\hat{V}(2 n)+\beta\left(n, z_{n}^{+}\right)\right)\left(\hat{V}(-2 n)+\beta\left(-n, z_{n}^{+}\right)\right)$, it follows that

$$
\zeta_{n}^{+}=\zeta_{n}^{-}=0 .
$$

For $n \in \mathcal{M}, G_{n}^{+}$and $G_{n}^{-}$are of the form

$$
G_{n}^{+}=x_{n}^{+} e^{-i n \pi x}+y_{n}^{+} e^{i n \pi x}+\sum_{k \neq \pm n} a_{x_{n}^{+}, y_{n}^{+}, z_{n}^{+}}(k) e^{i k \pi x},
$$




$$
G_{n}^{-}=x_{n}^{-} e^{-i n \pi x}+y_{n}^{-} e^{i n \pi x}+\sum_{k \neq \pm n} a_{x_{n}^{-}, y_{n}^{-}, z_{n}^{+}}(k) e^{i k \pi x},
$$

where $a_{x_{n}^{ \pm}, y_{n}^{ \pm}, z_{n}^{+}}$is given by $(2.56)$.

The normalization condition (3.3) leads to

$$
\begin{aligned}
x_{n}^{-}(1 & \left.+\sum_{k \neq \pm n}\left(z_{n}^{+}-B_{n}\right)^{-1}\left(\mathcal{S}^{n} \hat{V}\right)_{\mathbb{Z}(n)}(k)\right) \\
& +y_{n}^{-}\left(1+\sum_{k \neq \pm n}\left(z_{n}^{+}-B_{n}\right)^{-1}\left(\mathcal{S}^{n} \hat{V}\right)_{\mathbb{Z}(n)}(k)\right)=0,
\end{aligned}
$$

which, in view of Proposition 2.11, (iv), (v), yields, as $n \geq 2 n_{2}$ for $n \in \mathcal{M}$,

$$
\begin{aligned}
\left|x_{n}^{-}\right| & \leq\left|y_{n}^{-}\right|\left(1+\frac{1}{n}\|V\|\right) \frac{1}{1-\frac{1}{n}\|V\|} \\
& \leq\left|y_{n}^{-}\right|\left(1+\frac{4\|V\|}{n}\right) \leq 3\left|y_{n}^{-}\right|,
\end{aligned}
$$

and, similary,

$$
\left|y_{n}^{-}\right| \leq\left|x_{n}^{-}\right|\left(1+\frac{4\|V\|}{n}\right) \leq 3\left|x_{n}^{-}\right| .
$$

Using (3.14) and (3.15), one obtains from the normalization condition (3.3) the estimate

$$
\begin{aligned}
\frac{2}{3}\left|x_{n}^{-}\right|^{2} & \leq 2\left|x_{n}^{-} y_{n}^{-}\right|=\left|1-\sum_{k \neq \pm n} a_{x_{n}^{-}, y_{n}^{-}, z_{n}^{+}}(k) a_{x_{n}^{-}, y_{n}^{-}, z_{n}^{+}}(-k)\right| \\
& \leq 1+\left\|a_{x_{n}^{-}, y_{n}^{-}, z_{n}^{+}}\right\|^{2} \leq 1+\frac{\left|x_{n}^{-}\right|+\left|y_{n}^{-}\right|}{2} \frac{\|V\|}{n} \leq 1+2\left|x_{n}^{-}\right| \frac{\|V\|}{n} .
\end{aligned}
$$

It follows that for $n \geq 3 n_{2}$,

$$
\left|x_{n}^{-}\right| \leq \sqrt{\frac{16}{10}}+\frac{2}{3} \frac{\|V\|}{n} \leq 2 .
$$

Similarly, one can show that for $n \geq 3 n_{2}$

$$
\left|y_{n}^{-}\right| \leq \sqrt{\frac{16}{10}}+\frac{2}{3} \frac{\|V\|}{n} \leq 2 .
$$

Therefore, (3.13) leads to, using Proposition 2.11 (vi),

$$
\left|x_{n}^{-}+y_{n}^{-}\right| \leq \frac{4}{n}\|V\|,
$$

and (3.2) implies, for $n \geq 3 n_{2}$, using Proposition 2.11 (iii),

$$
\left|2 x_{n}^{-} y_{n}^{-}-1\right| \leq\left(\frac{4\|V\|}{n}\right)^{2} \leq \frac{4\|V\|}{n} .
$$

Estimates (3.18) and (3.19) imply, with (3.5), $n \in \mathcal{M}, n \geq 3 n_{2}$,

$$
\left|x_{n}^{-}-\frac{i}{\sqrt{2}}\right| \leq \frac{10\|V\|}{n} ; \quad\left|y_{n}^{-}+\frac{i}{\sqrt{2}}\right| \leq \frac{10\|V\|}{n} .
$$


Using (3.2) and (3.4) and the above estimates, we conclude, by similar computations, that, for $n \in \mathcal{M}$ with $n \geq 20 n_{2}$,

$$
\begin{aligned}
& \left|x_{n}^{+}\right| \leq 3, \quad\left|x_{n}^{+}-\frac{1}{\sqrt{2}}\right| \leq \frac{200\|V\|}{n} ; \\
& \left|y_{n}^{+}\right| \leq 3, \quad\left|y_{n}^{+}-\frac{1}{\sqrt{2}}\right| \leq \frac{200\|V\|}{n} .
\end{aligned}
$$

For $n \in \mathcal{M}, n \geq 20 n_{2}$, one obtains

$$
\begin{aligned}
\left(-\frac{d^{2}}{d x^{2}}+V-\tau_{n}\right) G_{n}^{+}= & \left(\lambda_{n}^{+}-\tau_{n}\right) G_{n}^{+}+\left(\hat{V}(-2 n)+\beta\left(-n, z_{n}^{+}\right)\right) y_{n}^{+} e^{-i n \pi x} \\
& +\left(\hat{V}(2 n)+\beta\left(n, z_{n}^{+}\right)\right) x_{n}^{+} e^{i n \pi x},
\end{aligned}
$$

which leads to

$$
\begin{aligned}
\left\langle G_{n}^{+},\right. & \left.\left(-\frac{d^{2}}{d x^{2}}+V-\tau_{n}\right) G_{n}^{+}\right\rangle \\
= & (\hat{V}(2 n)+\hat{V}(-2 n)) x_{n}^{+} y_{n}^{+}+x_{n}^{+} y_{n}^{+}\left(\beta\left(n, z_{n}^{+}\right)+\beta\left(-n, z_{n}^{+}\right)\right) \\
\quad= & \frac{1}{2}(\hat{V}(2 n)+\hat{V}(-2 n))+l_{N+1, \omega}^{2}(n)
\end{aligned}
$$

and

$$
\begin{aligned}
\left\langle G_{n}^{-},\right. & \left.\left(-\frac{d^{2}}{d x^{2}}+V-\tau_{n}\right) G_{n}^{+}\right\rangle \\
& =x_{n}^{-} y_{n}^{+} \hat{V}(-2 n)+y_{n}^{-} x_{n}^{+} \hat{V}(2 n)+x_{n}^{-} y_{n}^{+} \beta\left(-n, z_{n}^{+}\right)+y_{n}^{-} x_{n}^{+} \beta\left(n, z_{n}^{+}\right) \\
& =\frac{i}{2}(\hat{V}(-2 n)-\hat{V}(2 n))+l_{N+1, \omega}^{2}(n),
\end{aligned}
$$

where, by abuse of notation, we mean by $\left(l_{N+1, \omega}^{2}(n)\right)_{n \geq 1}$ an element in $l_{N+1, \omega}^{2}$ uniformly bounded for $V \in \mathcal{U}$, where $\mathcal{U}$ is a sufficiently small neighborhood of $V_{0} \in H_{0}^{N, \omega}\left(S^{1}, \mathbb{R}\right)$ in $H_{0}^{N, \omega}\left(S^{1} ; \mathbb{C}\right)$. Using the above estimates, one obtains

Lemma 3.2. Assume that $V_{0} \in H_{0}^{N, \omega}\left(S^{1}, \mathbb{R}\right)$. Then there exist a neighborhood $\mathcal{U}$ of $V_{0}$ in $H^{N, \omega}\left(S^{1} ; \mathbb{C}\right), n_{3} \geq n_{2}$ and $1 \leq C<\infty$ so that for $V \in \mathcal{U}$,

$$
\sum_{\substack{n \geq n_{3} \\ \lambda_{n}^{+}=\lambda_{n}^{-}}}(1+n)^{2 N+2} e^{2 n \omega}\left\|\Phi_{n}(V)-\underset{(\hat{V}(2 n)-\hat{V}(-2 n)) / 2 i}{(\hat{V}(2 n)+\hat{V}(-2 n)) / 2}\right\|^{2} \leq C .
$$

Let us now consider those $n \geq n_{2}$ with $\lambda_{n}^{+} \neq \lambda_{n}^{-}$. Denote by $f_{n}^{ \pm}$eigenfunctions of $\lambda_{n}^{ \pm}$normalized so that

$$
\sum_{k \in \mathbb{Z}} \hat{f}_{n}^{ \pm}(k)^{2}=1
$$

Then $G_{n}^{+}$and $G_{n}^{-}$are linear combinations of $f_{n}^{+}$and $f_{n}^{-}$,

$$
\begin{aligned}
G_{n}^{+} & =\alpha_{n}^{+} f_{n}^{+}+\alpha_{n}^{-} f_{n}^{-} \\
& =x_{n}^{+} e^{-i n \pi x}+y_{n}^{+} e^{i n \pi x}+\sum_{k \neq \pm n} b_{n}^{+}(k) e^{i k \pi x}
\end{aligned}
$$


with

$$
\begin{aligned}
b_{n}^{+}:= & \alpha_{n}^{+} \hat{f}_{n}^{+}(-n)\left(z_{n}^{+}-B_{n}\right)^{-1} \mathcal{S}^{n} \hat{V}+\alpha_{n}^{-} \hat{f}_{n}^{-}(-n)\left(z_{n}^{-}-B_{n}\right)^{-1} \mathcal{S}^{n} \hat{V} \\
& +\alpha_{n}^{+} \hat{f}_{n}^{+}(n)\left(z_{n}^{+}-B_{n}\right)^{-1} \mathcal{S}^{-n} \hat{V}+\alpha_{n}^{-} \hat{f}_{n}^{-}(n)\left(z_{n}^{-}-B_{n}\right)^{-1} \mathcal{S}^{-n} \hat{V}
\end{aligned}
$$

and

$$
\begin{aligned}
G_{n}^{-} & =\beta_{n}^{+} f_{n}^{+}+\beta_{n}^{-} f_{n}^{-} \\
& =x_{n}^{-} e^{-i n \pi x}+y_{n}^{-} e^{i n \pi x}+\sum_{k \neq \pm n} b_{n}^{-}(k) e^{i k \pi x}
\end{aligned}
$$

with $b_{n}^{-}$given by an expression similar to $b_{n}^{+}$.

For $V_{0} \in L_{0}^{2}\left(S^{1} ; \mathbb{R}\right)$ there exist a neighborhood $\mathcal{U}$ of $V_{0}$ in $L_{0}^{2}\left(S^{1} ; \mathbb{C}\right)$ and $n_{3} \geq n_{2}$ so that for $n \geq n_{3}$ (cf. [BBGK, Lemma 4.10], [Ka, Proposition 8])

$$
\begin{gathered}
x_{n}^{+}=\frac{1}{\sqrt{2}}+O\left(\frac{1}{n}\right), y_{n}^{+}=\frac{1}{\sqrt{2}}+O\left(\frac{1}{n}\right), \\
x_{n}^{-}=\frac{i}{\sqrt{2}}+O\left(\frac{1}{n}\right), y_{n}^{-}=-\frac{i}{\sqrt{2}}+O\left(\frac{1}{n}\right), \\
f_{n}^{+}(x)=\frac{e^{-i \theta_{n}}}{\sqrt{2}} e^{-i n \pi x}+\frac{e^{i \theta_{n}}}{\sqrt{2}} e^{i n \pi x}+O\left(\frac{1}{n}\right), \\
f_{n}^{-}(x)=i \frac{e^{-i \theta_{n}}}{\sqrt{2}} e^{-i n \pi x}-i \frac{e^{i \theta_{n}}}{\sqrt{2}} e^{i n \pi x}+O\left(\frac{1}{n}\right) .
\end{gathered}
$$

As $\lambda_{n}^{+} \neq \lambda_{n}^{-}$, the normalization conditions for $G_{n}^{ \pm}$imply

$$
\left(\alpha_{n}^{+}\right)^{2}+\left(\alpha_{n}^{-}\right)^{2}=1,\left(\beta_{n}^{+}\right)^{2}+\left(\beta_{n}^{-}\right)^{2}=1, \alpha_{n}^{+} \beta_{n}^{+}+\alpha_{n}^{-} \beta_{n}^{-}=0,
$$

which leads to

$$
\begin{aligned}
& \alpha_{n}^{+}=\frac{e^{i \theta_{n}}+e^{-i \theta_{n}}}{2}+O\left(\frac{1}{n}\right), \alpha_{n}^{-}=\frac{e^{i \theta_{n}}-e^{-i \theta_{n}}}{2 i}+O\left(\frac{1}{n}\right), \\
& \beta_{n}^{+}=-\frac{e^{i \theta_{n}}-e^{-i \theta_{n}}}{2 i}+O\left(\frac{1}{n}\right), \beta_{n}^{-}=\frac{e^{i \theta_{n}}+e^{-i \theta_{n}}}{2}+O\left(\frac{1}{n}\right) .
\end{aligned}
$$

When written in Fourier space, $\left(-\frac{d^{2}}{d x^{2}}+V-\tau_{n}\right) G_{n}^{+}$takes the form (cf. (2.3))

$$
\left(\begin{array}{c}
-x_{n}^{+}\left(z_{n}^{+}+z_{n}^{-}\right) / 2+y_{n}^{+} \hat{V}(-2 n)+\left\langle\mathcal{S}^{n} \mathcal{J} \hat{V}, b_{n}^{+}\right\rangle \\
x_{n}^{+} \hat{V}(2 n)-y_{n}^{+}\left(z_{n}^{+}+z_{n}^{-}\right) / 2+\left\langle\mathcal{S}^{-n} \mathcal{J} \hat{V}, b_{n}^{+}\right\rangle \\
x_{n}^{+} \mathcal{S}^{n} \hat{V}+y_{n}^{+} \mathcal{S}^{-n} \hat{V}+\left(B_{n}-\left(z_{n}^{+}+z_{n}^{-}\right) / 2\right) b_{n}^{+}
\end{array}\right) .
$$


The terms appearing in this expression are discussed separately. Since $\alpha(n, z)=$ $\alpha(-n, z)$, one obtains

$$
\begin{aligned}
\left\langle\mathcal{S}^{n} \mathcal{J V}, b_{n}^{+}\right\rangle= & \alpha_{n}^{+} \hat{f}_{n}^{+}(-n) \alpha\left(n, z_{n}^{+}\right)+\alpha_{n}^{-} \hat{f}_{n}^{-}(-n) \alpha\left(n, z_{n}^{-}\right) \\
& +\alpha_{n}^{+} \hat{f}_{n}^{+}(n) \beta\left(-n, z_{n}^{+}\right)+\alpha_{n}^{-} \hat{f}_{n}^{-}(n) \beta\left(-n, z_{n}^{-}\right) \\
= & x_{n}^{+} \frac{\alpha\left(n, z_{n}^{+}\right)+\alpha\left(n, z_{n}^{-}\right)}{2}+x_{n}^{+} \frac{\alpha\left(n, z_{n}^{+}\right)-\alpha\left(n, z_{n}^{-}\right)}{2} \\
& +\alpha_{n}^{-} \hat{f}_{n}^{-}(-n)\left(\alpha\left(n, z_{n}^{+}\right)-\alpha\left(n, z_{n}^{-}\right)\right) \\
& +\alpha_{n}^{+} \hat{f}_{n}^{+}(n) \beta\left(-n, z_{n}^{+}\right)+\alpha_{n}^{-} \hat{f}_{n}^{-}(n) \beta\left(-n, z_{n}^{-}\right) .
\end{aligned}
$$

Taking into account that $-\zeta_{n}^{ \pm}=-z_{n}^{ \pm}+\alpha\left(n, z_{n}^{ \pm}\right)$and thus

$$
-\frac{z_{n}^{+}+z_{n}^{-}}{2}+\frac{\alpha\left(n, z_{n}^{+}\right)+\alpha\left(n, z_{n}^{-}\right)}{2}=-\frac{\zeta_{n}^{+}+\zeta_{n}^{-}}{2}
$$

the first component in $(3.28)$ can be written as

$$
-x_{n}^{+}\left(\frac{z_{n}^{+}+z_{n}^{-}}{2}\right)+y_{n}^{+} \hat{V}(-2 n)+\left\langle\mathcal{S}^{n} \mathcal{J} \hat{V}, b_{n}^{+}\right\rangle=\hat{V}(-2 n) y_{n}^{+}+g_{n}^{+}(-n)
$$

where

$$
\begin{aligned}
g_{n}^{+}(-n)= & \left(x_{n}^{+}+\alpha_{n}^{-} \hat{f}_{n}^{-}(-n)\right)\left(\alpha\left(n, z_{n}^{+}\right)-\alpha\left(n, z_{n}^{-}\right)\right)-x_{n}^{+} \frac{\zeta_{n}^{+}+\zeta_{n}^{-}}{2} \\
& +\alpha_{n}^{+} \hat{f}_{n}^{+}(n) \beta\left(-n, z_{n}^{+}\right)+\alpha_{n}^{-} \hat{f}_{n}^{-}(n) \beta\left(-n, z_{n}^{-}\right) .
\end{aligned}
$$

In view of (3.24)-(3.27), Lemma 2.4(ii), Proposition 2.7 and Theorem 2.10 we conclude that $g_{n}^{+}(-n)=l_{N+1, \omega}^{2}(n)$, uniformly bounded for $V$ in a sufficiently small neighborhood $\mathcal{U}$ of $V_{0}$ in $H_{0}^{N, \omega}\left(S^{1}, \mathbb{C}\right)$.

The second component in (3.28) is analyzed similarly, to yield

$$
x_{n}^{+} \hat{V}(2 n)-y_{n}^{+}\left(z_{n}^{+}+z_{n}^{-}\right) / 2+\left\langle\mathcal{S}^{-n} \mathcal{J} \hat{V}, b_{n}^{+}\right\rangle=\hat{V}(2 n) x_{n}^{+}+g_{n}^{+}(n)
$$

where

$$
\begin{aligned}
g_{n}^{+}(n)= & \left(-y_{n}^{+}+\alpha_{n}^{+} \hat{f}_{n}^{+}(n)\right) \frac{\alpha\left(n, z_{n}^{+}\right)-\alpha\left(n, z_{n}^{-}\right)}{2}-y_{n}^{+} \frac{\zeta_{n}^{+}+\zeta_{n}^{-}}{2} \\
& +\alpha_{n}^{+} \hat{f}_{n}^{+}(-n) \beta\left(n, z_{n}^{+}\right)+\alpha_{n}^{-} \hat{f}_{n}^{-}(-n) \beta\left(n, z_{n}^{-}\right)
\end{aligned}
$$

and we again conclude that $g_{n}^{+}(-n)=l_{N+1, \omega}^{2}(n)$.

Finally we analyze the third component in (3.28): 
We compute (with $\gamma_{n}:=\lambda_{n}^{+}-\lambda_{n}^{-}$)

$$
\begin{aligned}
\left(B_{n}-\frac{z_{n}^{+}+z_{n}^{-}}{2}\right) b_{n}^{+}= & -\left(\alpha_{n}^{+} \hat{f}_{n}^{+}(-n)+\alpha_{n}^{-} \hat{f}_{n}^{-}(-n)\right) \mathcal{S}^{n} \hat{V} \\
& -\left(\alpha_{n}^{+} \hat{f}_{n}^{+}(n)+\alpha_{n}^{-} \hat{f}_{n}^{-}(-n)\right) \mathcal{S}^{-n} \hat{V} \\
& +\alpha_{n}^{+} \hat{f}_{n}^{+}(-n) \frac{\gamma_{n}}{2}\left(z_{n}^{+}-B_{n}\right)^{-1} \mathcal{S}^{n} \hat{V} \\
& +\alpha_{n}^{-} \hat{f}_{n}^{-}(-n) \frac{\gamma_{n}}{2}\left(z_{n}^{-}-B_{n}\right)^{-1} \mathcal{S}^{n} \hat{V} \\
& +\alpha_{n}^{+} \hat{f}_{n}^{+}(n) \frac{\gamma_{n}}{2}\left(z_{n}^{+}-B_{n}\right)^{-1} \mathcal{S}^{-n} \hat{V} \\
& +\alpha_{n}^{-} \hat{f}_{n}^{-}(n) \frac{\gamma_{n}}{2}\left(z_{n}^{-}-B_{n}\right)^{-1} \mathcal{S}^{-n} \hat{V} .
\end{aligned}
$$

Thus

$$
\begin{aligned}
x_{n}^{+} \mathcal{S}^{n} \hat{V} & +y_{n}^{+} \mathcal{S}^{-n} \hat{V}+\left(B_{n}-\frac{z_{n}^{+}+z_{n}^{-}}{2}\right) b_{n}^{+} \\
= & \alpha_{n}^{+} \hat{f}_{n}^{+}(-n) \frac{\gamma_{n}}{2}\left(z_{n}^{+}-B_{n}\right)^{-1} \mathcal{S}^{n} \hat{V} \\
& +\alpha_{n}^{-} \hat{f}_{n}^{-}(-n) \frac{\gamma_{n}}{2}\left(z_{n}^{-}-B_{n}\right)^{-1} \mathcal{S}^{n} \hat{V} \\
& +\alpha_{n}^{+} \hat{f}_{n}^{+}(n) \frac{\gamma_{n}}{2}\left(z_{n}^{+}-B_{n}\right)^{-1} \mathcal{S}^{-n} \hat{V} \\
& +\alpha_{n}^{-} \hat{f}_{n}^{-}(n) \frac{\gamma_{n}}{2}\left(z_{n}^{-}-B_{n}\right)^{-1} \mathcal{S}^{-n} \hat{V} \\
= & l_{N+1, \omega}^{2}(n) .
\end{aligned}
$$

Combining (3.30)-(3.34), we obtain, for $n \geq n_{3}$ with $\lambda_{n}^{+} \neq \lambda_{n}^{-}$,

$$
\begin{aligned}
\left\langle G_{n}^{+},\left(-\frac{d^{2}}{d x^{2}}+V-\tau_{n}\right) G_{n}^{+}\right\rangle & =\hat{V}(-2 n)\left(y_{n}^{+}\right)^{2}+\hat{V}(2 n)\left(x_{n}^{+}\right)^{2}+l_{N+1, \omega}^{2}(n), \\
\left\langle G_{n}^{-},\left(-\frac{d^{2}}{d x^{2}}+V-\tau_{n}\right) G_{n}^{+}\right\rangle & =\hat{V}(-2 n) x_{n}^{-} y_{n}^{+}+\hat{V}(2 n) y_{n}^{-} x_{n}^{+}+l_{N+1, \omega}^{2}(n) .
\end{aligned}
$$

In view of $\left(3.24^{ \pm}\right)$one then concludes the following:

Lemma 3.3. Assume $V_{0} \in H^{N, \omega}\left(S^{1} ; \mathbb{R}\right)$. Then there exist a neighborhood $\mathcal{U}$ of $V_{0}$ in $H^{N, \omega}\left(S^{1} ; \mathbb{C}\right), n_{3} \geq n_{2}$ and $1 \leq C<\infty$ such that for any $V \in \mathcal{U}$ and $n \geq n_{3}$

$$
\sum_{\substack{n \geq n_{3} \\ \lambda_{n}^{+} \neq \lambda_{n}^{-}}}(1+n)^{2 N+2} e^{2 n \omega}\left\|\Phi_{n}(V)-\left(\frac{\hat{V}(2 n)+\hat{V}(-2 n)}{2}\right)\right\|^{2} \leq C .
$$

Proposition 3.4. The map $\Phi^{(N, \omega)}: H_{0}^{N, \omega}\left(S^{1}\right) \rightarrow l_{N, \omega}^{2}\left(\mathbb{R}^{2}\right)$ has the following properties:

(i) $\Phi^{(N, \omega)}$ is bijective and real analytic; 
(ii) $\left(\Phi^{(N, \omega)}\right)^{-1}$ is real analytic;

Proof. As $\Phi^{(N, \omega)}=\left.\Phi\right|_{H_{0}^{N, \omega}\left(S^{1}\right)}$ and $\Phi: L_{0}^{2}\left(S^{1}\right) \rightarrow l^{2}\left(\mathbb{R}^{2}\right)$ is bijective and bianalytic, we conclude that $\Phi^{(N, \omega)}$ is one-to-one and, for $V \in H_{0}^{N}\left(S^{1}\right), d_{V} \Phi^{(N, \omega)}$ is also one-to-one. From Corollary 3, stated in the introduction, and the fact that $\Phi$ is onto we conclude that $\Phi^{(N, \omega)}$ is onto. By Proposition 3.1, $\Phi^{(N, \omega)}$ is real analytic. To prove statement (ii), it suffices to show that $d_{V} \Phi^{(N, \omega)}$ is onto for an arbitrary element $V$ in $H_{0}^{N, \omega}\left(S^{1}\right)$. By (i) and the Fredholm alternative it suffices to prove that $d_{V} \Phi^{(N, \omega)}=A+K$, where $A: H_{0}^{N, \omega}\left(S^{1}\right) \rightarrow l_{N, \omega}^{2}\left(\mathbb{R}^{2}\right)$ is a linear isomorphism and $K$ is a compact operator. This follows from Proposition 3.1.

Next we consider the map $\Lambda: L_{0}^{2}\left(S^{1}\right) \rightarrow l_{1 / 2}^{2}\left(\mathbb{R}^{2}\right)$, defined in [BBGK] by $\Lambda(V)=$ $\left(\Lambda_{n}(V)\right)_{n \geq 1}$ with $\Lambda_{n}(V)=\xi_{n}(V) \Phi_{n}(V)$. Here

$$
\xi_{n}(V)^{2}=\frac{2 \mathcal{I}_{n}(V)}{\gamma_{n}(V) / 2}
$$

and $\mathcal{I}_{n}(V)(n \geq 1)$ denote the action variables of $\mathrm{KdV}$ with respect to the Gardner bracket ([FM]; cf. [BBGK])

$$
\mathcal{I}_{n}(V)=\frac{2}{\pi} \int_{\lambda_{2 n-1}}^{\lambda_{2 n}} \mu \frac{\dot{\triangle}(\mu)}{(\triangle(\mu)-4)^{1 / 2}} d \mu .
$$

Define $\Lambda^{(N, \omega)}:=\left.\Lambda\right|_{H_{0}^{N, \omega}{ }_{\left(S^{1}\right)}}$. From the asymptotics, valid uniformly on sets of potentials bounded in $L_{0}^{2}\left(S^{1} ; \mathbb{C}\right)$.

$$
\xi_{n}=\frac{1}{\sqrt{n \pi}}\left(1+O\left(\frac{\log n}{n}\right)\right)
$$

(cf. [BBGK]) we conclude that $\Lambda^{(N, \omega)}\left(H_{0}^{N, \omega}\left(S^{1}\right)\right) \subset l_{N+\frac{1}{2}, \omega}^{2}\left(\mathbb{R}^{2}\right)$.

Proposition 3.5. The map $\Lambda^{(N, \omega)}: H_{0}^{N, \omega}\left(S^{1}\right) \rightarrow l_{N+\frac{1}{2}, \omega}^{2}\left(\mathbb{R}^{2}\right)$ has the following properties:

(i) $\Lambda^{(N, \omega)}$ is bijective and real analytic;

(ii) $\left(\Lambda^{(N, \omega)}\right)^{-1}$ is real analytic;

(iii) for any $0 \leq \varepsilon<1 / 2$,

$$
\Lambda_{n}^{(N, \omega)}(V)=\frac{1}{\sqrt{n \pi}}\left(\frac{\hat{V}(2 n)+\hat{V}(-2 n)}{2}, \frac{\hat{V}(2 n)-\hat{V}(-2 n)}{2 i}\right)+l_{N+1+\varepsilon, \omega}^{2}(n)
$$

uniformly on sets of potentials bounded in $H_{0}^{N, \omega}\left(S^{1} ; \mathbb{C}\right)$.

Proof. We argue as in the proof of Proposition 3.4 to conclude from [BBGK, section $2]$ that $\Lambda^{(N, \omega)}$ is bijective and real analytic and that $d_{V} \Lambda^{(N, \omega)}$ is one-to-one for any $V \in H_{0}^{N, \omega}\left(S^{1}\right)$. Statement (iii) follows from (3.35) and Proposition 3.1. To prove statement (ii) it suffices to prove that $d_{V} \Lambda^{(N, \omega)}$ is onto for any $V$ in $H_{0}^{N, \omega}\left(S^{1}\right)$.

Write

$$
d_{V} \Lambda_{n}(W)=\xi_{n}(V) d_{V} \Phi_{n}(W)+d_{V} \xi_{n}(W) \Phi_{n}(V)
$$

and introduce, for $V$ fixed,

$$
A:=\left(A_{n}\right)_{n \geq 1}: H_{0}^{N, \omega}\left(S^{1}\right) \rightarrow l_{N+\frac{1}{2}, \omega}^{2}\left(\mathbb{R}^{2}\right)
$$


with $A_{n}(W):=\frac{1}{\sqrt{n \pi}} d_{V} \Phi_{n}(W)$. By Proposition 3.4, $A$ is a linear isomorphism. Moreover, by [BBGK, Lemma 4.18], for any $0 \leq \varepsilon<1 / 2$,

$$
d_{V} \xi_{n}(W)=O\left(\frac{1}{n^{1+\varepsilon}}\right)\|W\|_{L^{2}} .
$$

Substituting (3.35) and (3.37) into (3.36), we conclude that, for any $0 \leq \varepsilon<1 / 2$, there exists $C_{\varepsilon}>0$ such that

$$
\left\|\left(d_{V} \Lambda_{n}(W)-A_{n}(W)\right)_{n \geq 1}\right\|_{l_{N+1+\varepsilon, \omega}^{2}} \leq C_{\varepsilon}\|W\|_{L^{2}} .
$$

Estimate (3.21) implies that $d_{V} \Lambda^{(N, \omega)}=A+K$, where $K$ is compact. By the Fredholm alternative and the fact that $d_{V} \Lambda^{(N, \omega)}$ is one-to-one, we conclude that $d_{V} \Lambda^{(N, \omega)}$ is onto. This implies statement (ii).

Proof of Theorem 1. Theorem 1 follows from Proposition 3.5.

\section{REFERENCES}

[BBGK] D. Bättig, A. Bloch, J.-C. Guillot, T. Kappeler; On the symplectic structure of the phase space for periodic KdV, Toda and defocusing NLS, Duke Math. J. 79 (1995), 549-604. MR 96i: 58065

[BKM1] D. Bättig, T. Kappeler, B. Mityagin, On the Korteweg-de Vries equation: convergent Birkhoff normal form, J. Funct. Anal. 140 (1996), 335-358. MR 97g:58073

[BKM2] D. Bättig, T. Kappeler, B. Mityagin, On the Korteweg-de Vries equation: frequencies and initial value problem, Pacific J. Math. 181 (1997), 1-55. CMP 98:06

[Bo] J. Bourgain, Fourier transform restriction phenomena for certain lattice subsets and applications to nonlinear evolution equations, part II: KdV-equation, Geom. Funct. Anal. 3 (1993), 209-262. MR 95b:35160b

[DKN] B.A. Dubrovin, I.M. Krichever, S.P. Novikov, Integrable systems I, in Dynamical Systems IV, ed. V.I. Arnold, S.P. Novikov, Encycl. of Math. Sci., Springer Verlag, 1990. MR 87k:58112

[FM] H. Flaschka, D. McLaughlin, Canonically conjugate variables for the Korteweg-de Vries equation and the Toda lattice with periodic boundary conditions, Progress of Theor. Phys. 55 (1976), 438-456. MR 53:7179

[Ka] T. Kappeler, Fibration of the phase-space for the Korteweg-de Vries equation, Ann. Inst. Fourier 41 (1991), 539-575. MR 92k:58212

[Ma] V.A. Marčenko, Sturm-Liouville Operators and Applications, Birkhäuser, Basel, 1986. MR 88f: 34034

[MT1] H. P. McKean, E. Trubowitz, Hill's operator and hyperelliptic function theory in the presence of infinitely many branch points, Comm. Pure Appl. Math. 24 (1976), 143226. MR 55:761

[MT2] H.P. McKean, E. Trubowitz, Hill's surfaces and their theta functions, Bull. AMS, 84 (1978), 1042-1085. MR 80b:30039

[PT] J. Pöschel, E. Trubowitz, Inverse Spectral Theory, Academic Press, 1987. MR 89b:34061

Institut Für Mathematik, Universität Zürich, Winterthurerstrasse 190, CH-8057 ZüRICH, SWITZERLAND

E-mail address: tk@math.unizh.ch

Department of Mathematics, Ohio State University, Columbus, Ohio 43210

E-mail address: borismit@math.ohio-state.edu 